\title{
Emergence and evolution of tripole vortices from net-circulation initial conditions
}

\author{
L. A. Barba \\ Department of Mathematics, University of Bristol, Bristol BS8 1TW, United Kingdom \\ A. Leonard \\ Graduate Aeronautical Laboratories, California Institute of Technology, Pasadena, California 91125
}

(Received 13 April 2006; accepted 16 November 2006; published online 3 January 2007)

\begin{abstract}
The emergence of coherent vortical structures is a hallmark of the evolution of two-dimensional turbulence. Two fundamental processes of this evolution have been identified in vortex merging and vortex axisymmetrization. The question of whether axisymmetrization is a universal process has recently been answered in the negative. In the linear approximation, vortices indeed become axisymmetric, due to shear-enhanced diffusion. In the case of nonlinear interactions, other outcomes are possible; in the present work, we discuss a situation in which the flow reorganizes into a tripolar vortex. By performing an extensive numerical study, spanning the parameter space, we pursue the questions of what dictates if the flow will become axisymmetric or will develop into a quasisteady tripolar vortex, and what are the stages and the time scales of the flow evolution. The initial condition in this study consists of a Gaussian monopole with a quadrupolar perturbation. The amplitude of the perturbation and the Reynolds number determine the evolution. A tripole emerges for sufficiently large amplitude of the perturbation, and we seek to find a critical amplitude that varies with Reynolds number. We make several physical observations derived from visualizing and postprocessing numerous flow simulations: looking at the decay of the perturbation with respect to viscous or shear diffusion time scales; applying mixing theory; obtaining the first few azimuthal modes of the vorticity field; and describing the long-time evolution. () 2007 American Institute of Physics. [DOI: 10.1063/1.2409734]
\end{abstract}

\section{INTRODUCTION}

The emergence, behavior, and persistence of vortices in two-dimensional flows has for long been a subject of fascination to fluid dynamicists. Particularly in the last two decades, since the observation of vorticity concentrations that arise spontaneously from random initial conditions, ${ }^{1}$ a huge interest in these "coherent structures" of turbulence has developed. The behavior of coherent vortices is characterized by their tendency to assume axisymmetric shapes, their stability, and their evolution in a cascade to larger scales.

The relaxation towards axisymmetry has been discussed intensely. It was first detected in the evolution of cascading two-dimensional (2D) turbulence, ${ }^{1}$ occurring systematically after vortex interactions, and then it was argued to be a generic feature of two-dimensional vortices. ${ }^{2}$ That is, it was implied that all localized and smooth concentrations of vorticity will naturally approach axisymmetry.

The calculations of Melander $e t a l .{ }^{2}$ use smooth elliptical vortices as initial conditions, and establish that these behave very differently from their sharp counterpart, the Kirchhoff vortex. The latter is an exact solution of the Euler equations, consisting in an elliptical patch of constant vorticity which rotates steadily without change of shape (Ref. 3, Art. 159). It might seem plausible that the smoothed vortices should behave nearly in the same way, perhaps slowly becoming axisymmetric on a diffusion time scale. The results of Melander et $a l^{2}$ show, however, that axisymmetrization occurs quite quickly and that it is an inviscid process. The kinematics of the process was then comprehensively explained by and attributed to the formation and evolution of filaments from the tips of the elliptical compact vortices. Filamentation, it was argued, brings upon a deviation from the elliptical symmetry, which in turn activates a so-called "axisymmetrization principle," relating changes in aspect ratio to the relative orientations of vorticity contours and streamlines.

This result, together with demonstrations of stability for axisymmetric flows under certain conditions, ${ }^{4}$ led to suggestions that axisymmetric vortices may be attracting states in $2 \mathrm{D}$ flows at large Reynolds number. ${ }^{5}$ By means of the WKB approximation, Bernoff and Lingevitch ${ }^{5}$ determined that rapid variations of vorticity decay on a fast time scale in the order of $\mathrm{Re}^{1 / 3}$, the shear-diffusion time scale ${ }^{6}$ of passive scalars. The assumption of rapid variation is justified by the creation of small scales due to spiral wind-up of the perturbation in the base vortex. Hence, they show that an axisymmetric, planar vortex returns to axisymmetry after being perturbed, and it does so at the shear-diffusion time scale (i.e., faster than diffusion). Lundgren ${ }^{7}$ had previously arrived at this result by different means, developing asymptotic 2D spiral solutions. In the viscous case, and under the assumptions of large time and small viscosity $\nu$, Lundgren shows that the characteristic decay time for all the higher harmonics is proportional to $\nu^{-1 / 3}$. Only the axially symmetric component of the flow decays on the slower time scale proportional to $\nu^{-1}$.

It may seem suspect to claim that the shear-diffusion mechanism which homogenizes a passive scalar in a vortex 
acts in an identical fashion to wane vorticity perturbations. After all, vorticity is dynamically coupled to the velocity/ streamfunction. Lundgren argues that as nonaxisymmetric perturbations become rapidly varying radially, through the effect of differential rotation, the coupling of streamfunction and vorticity cancels at leading order. Bernoff and Lingevitch $^{5}$ also rely on the assumption of rapid variation, and show that far from the core of the vortex the perturbation streamfunction becomes exponentially small. They then address the assumption by numerically observing this rapid winding up of a perturbation in a Lamb vortex. The numerical validation, however, relies on the linearized disturbance equations.

In brief, a perturbed smooth vortex relaxes and becomes axisymmetric in the fast shear-diffusion time scale, within the context of linear theory. Indeed, the linear approximation results in an equation for the perturbation vorticity which is exactly the advection-diffusion equation except for a term that becomes exponentially small far from the core of the base vortex. Close to the core, on the other hand, the perturbation becomes rapidly varying due to the shearing caused by differential rotation. The perturbation thus becomes stretched to very small scales, where diffusion rapidly acts to homogenize it. As this occurs, the perturbation is said to be "expelled" to larger radii, where the streamfunction coupling is negligible, and the vorticity perturbation behaves like a passive scalar (see Bernoff and Lingevitch ${ }^{5}$ ).

The relaxation to axisymmetry in the inviscid case was analyzed by Bassom and Gilbert, ${ }^{8}$ by means of both asymptotics and numerical studies. In the absence of viscosity, the vorticity perturbations continue to cascade to small scales due to wind-up, and are never completely diffused. The process of axisymmetrization is understood in a coarse-grained sense in this context; i.e., the nonaxisymmetric perturbation tends to zero on average. These studies were limited to sufficiently smooth vortices, in the linear approximation.

Full nonlinear simulations of the same flow as used by Bernoff and Lingevitch, ${ }^{5}$ consisting in a Lamb vortex with an $m=2$ perturbation, produced conspicuously different results than in the linear case. ${ }^{9}$ It was found that, if the amplitude of the perturbation is large enough, the flow does not relax to axisymmetry. Instead, one observes the appearance of a quasisteady, rotating tripole which seems to evolve in the viscous time scale. Rossi et al. ${ }^{9}$ suggest that there is a threshold amplitude below which the flow evolves toward axisymmetry. Above the threshold, they argue, the negative portion of the $m=2$ perturbation does not become mixed, due to the creation of a separatrix of the streamlines in a frame rotating with the structure. In contrast, the test flows of Melander et al. ${ }^{2}$ leading to the suggestion of universality of axisymmetry, involved purely positive vorticity distributions. Without negative vorticity, the shear-diffusion effects were not disrupted. Clearly, if the perturbation vorticity has a considerable effect in the streamfunction, then the sheardiffusion mechanism can fail.

Other investigations have also suggested that axisymmetrization is not a universal process. Simulations using contour dynamics methods with contour surgery (see next section) by Dritschel ${ }^{10}$ show an elliptical vortex which evolves to a nonaxisymmetric structure consisting of an elliptical core surrounded by weak filaments. Some experimental results in magnetically confined plasmas have exhibited similar nonaxisymmetric asymptotic states. ${ }^{11}$ Koumoutsakos ${ }^{12}$ studied numerically, with a high-resolution vortex method, the inviscid evolution of several vorticity profiles. This study suggested that axisymmetrization occurs for smooth vortices, whereas nonaxisymmetric configurations result from sharp vortices. Further studies using a generic class of vortices with sharp edges ${ }^{13}$ indicated that the steepness of the vortex determines the degree of its axisymmetrization. Since inviscid vortices tend to have sharp edges due to "stripping,", "14,15 the implication is that, in the absence of viscosity, vortices in general do not tend towards axisymmetry. Viscosity would thus be a contributing factor to axisymmetrization, because it has the effect of smoothing sharp edges.

In the present paper, we continue the discussion of axisymmetrization (or lack thereof) of planar vortices. Using the Lamb-Oseen vortex with a superposed $m=2$ perturbation of moderate amplitude, a numerical study is performed spanning the parameter space given by $\delta$ and Re, the amplitude of perturbation and the Reynolds number, respectively. Rossi et $a l{ }^{9}$ gave results for $\delta=0.25$ and $\operatorname{Re}=10^{3}, 5 \times 10^{3}, 10^{4}$, which we have reproduced. In addition, we present calculations with varying perturbation amplitudes, and attempt to determine whether there is in fact a threshold amplitude that separates two asymptotic states (axisymmetric and tripolar). The possible relationship between this threshold amplitude and Reynolds number is sought, and several observations are made regarding the nonlinear evolution of the perturbed vortex.

\section{NUMERICAL METHOD}

In the numerical study of two dimensional vortical flows, the preferred methods of computation seem to be contour dynamics methods and pseudospectral methods. Contour dynamics, introduced by Zabusky et al. ${ }^{16}$ consists in a Lagrangian scheme that calculates the inviscid evolution of vorticity jumps in flows consisting of piecewise constant distributions of vorticity. They suffer the problem of production of finer and finer structures, due to filamentation, but this has been addressed by the variant called contour surgery. ${ }^{10,17}$

For applications in viscous vortex interactions and turbulence, many workers use the pseudospectral methods. This is the case in the studies of Melander $\mathrm{et} a \mathrm{l}^{2}$ that put forth the axisymmetrization principle. Most pseudospectral calculations use hyperviscosity; it has been suggested, however, that the hyperviscosity operator may introduce spurious dynamics. ${ }^{18}$ In studies of vortex stripping with combined strain and diffusion effects, ${ }^{19}$ it was found to introduce unphysical numerical artifacts. On the other hand, the standard spectral method calculates a periodic domain and often a large computational box is needed for simulation of an unbounded vortex.

Presently, we use a two-dimensional viscous vortex blob method. ${ }^{20}$ An inviscid vortex method was used in the work of Koumoutsakos ${ }^{12}$ on elliptical vortices, whereas Rossi et $a l .{ }^{9}$ used a viscous method based on core spreading, de- 


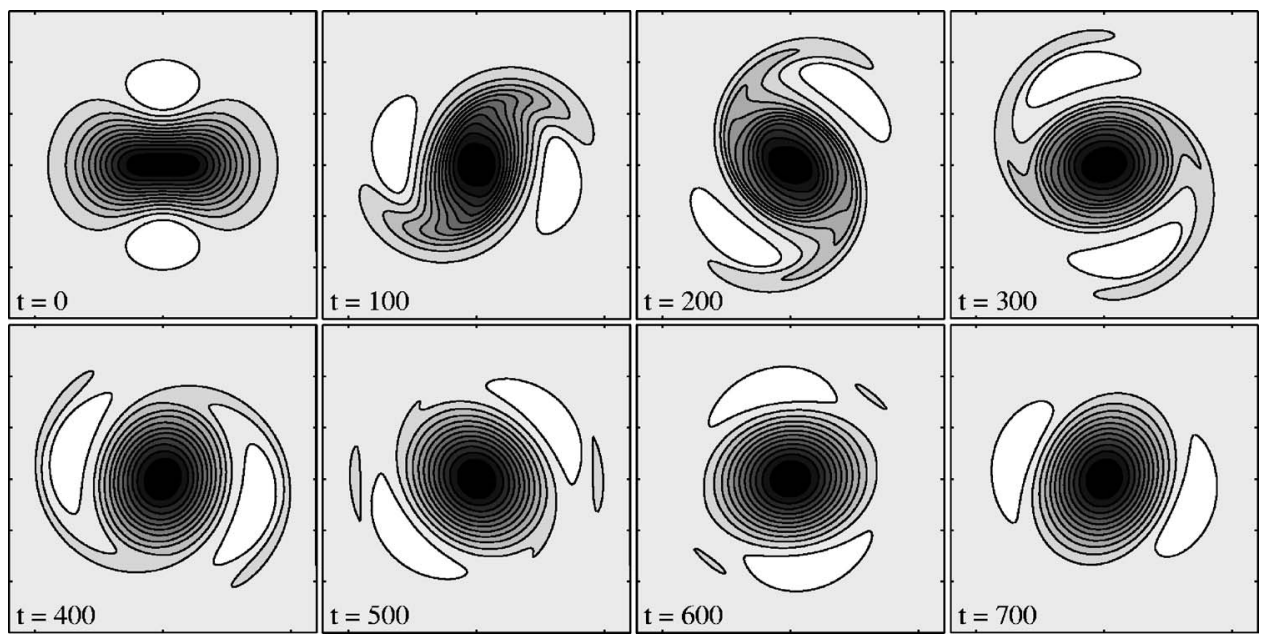

FIG. 1. Initial condition of Eqs. (1) and (2) relaxing to a quasisteady tripole; $\operatorname{Re}=10^{4}, \quad \delta=0.25 ; 14$ equally spaced contours of vorticity normalized by $\omega_{\max }(0)$. scribed by Rossi. ${ }^{21}$ The method used herein also utilizes the core spreading approach to viscosity, but applies a new concept of meshless spatial adaption; the method was fully tested and presented by $\mathrm{Barba}^{22}$ and Barba et al. ${ }^{23}$

The vortex method was implemented in parallel, using the PETSc library. ${ }^{24}$ The calculations presented here were carried out in 8-12 processors of a Beowulf cluster, and the number of vortex particles was in the order of $10^{4}$. The current limitation is mainly one of memory, as a square matrix of rank equal to the number of particles is built on initialization and upon each process of spatial adaption. As described in detail by $\mathrm{Barba}^{22}$ and Barba et al., ${ }^{23}$ the method utilizes radial basis function interpolation to find the circulation strength of the vortex particles on initialization and after spatial adaption. Radial basis function interpolation is a highly accurate method for interpolation of scattered data, ${ }^{25-27}$ but it requires the solution of a global influence problem. Preconditioned iterative methods are thus necessary, ${ }^{28}$ and presently these were provided within the PETSc library. In the future, the efficiency of the implementation can be improved by using a matrix-free approach, which is possible in PETSc using the "matrix shell" object. PETSc is an advanced library for large-scale scientific computation, and provides all the functionality needed for the parallel vector and matrix assembly and most operations, doing most of the message passing so that the codes have a minimal amount of direct calls to message passing interface functions.

The numerical parameters used in these computations were: an initial spacing of the particles $h=0.15$, and sometimes 0.12 or 0.096 (the particles are placed on nodes of a triangular lattice); an initial overlap of particles $h / \sigma=0.8$, where $\sigma$ stands for the core radius of the vortex blobs. The core basis function is a Gaussian: $\left(2 \pi \sigma^{2}\right)^{-1} \exp \left(-r^{2} / 2 \sigma^{2}\right)$. The time step is $\Delta t=0.5$ and integration is performed via fourth-order Runge-Kutta; frequency of spatial adaption is every ten time steps (resulting in a maximum core size due to core spreading of 0.1901 for $\mathrm{Re}=10^{4}$, and 0.2125 for $\mathrm{Re}$ $=10^{3}$, for the coarsest case where $h=0.15$ and $\sigma=0.1875$ ). The above parameters relate to a base vortex that has total circulation equal to 1.0, and a Gaussian radius equal to 2.0. For details of accuracy studies with the present vortex method, see Refs. 22 and 29.

\section{EMERGENCE OF A TRIPOLE FROM PERTURBED VORTICES}

The flow under study consists of a Gaussian vortex with a superposed $m=2$ (quadrupolar) perturbation that can be of order 1 . The initial condition is given by $\omega=\omega_{o}+\omega^{\prime}$, with

$$
\begin{aligned}
& \omega_{o}(\mathbf{x})=\frac{1}{4 \pi} \exp \left(\frac{-|\mathbf{x}|^{2}}{4}\right), \\
& \omega^{\prime}(\mathbf{x})=\frac{\delta}{4 \pi}|\mathbf{x}|^{2} \exp \left(\frac{-|\mathbf{x}|^{2}}{4}\right) \cos m \theta,
\end{aligned}
$$

where $\omega_{o}$ stands for the base vorticity, $\omega^{\prime}$ for the perturbation, and $\theta=\arg (\mathbf{x})$. The main case discussed in Rossi et al. ${ }^{9}$ corresponds to $\delta=0.25$ and $\operatorname{Re}=10^{4}$, with $\operatorname{Re}=\Gamma / \nu$ (total circulation divided by the viscosity). Figure 1 shows the evolution of the total vorticity for this case. (See Barba et al. ${ }^{23}$ for a comparison of results with our method to the results of Rossi et al., ${ }^{9}$ using similar line-contour plots of total vorticity and perturbation vorticity.)

The appearance of the tripole in the simulation depicted in Fig. 1 is significant for several reasons. The tripolar vortex is much less common than the monopole and the dipole in numerical and experimental observations. A notable experiment in which a tripole emerged from an unstable vortex in rotating flow is that of van Heijst and Kloosterziel. ${ }^{30}$ At the time, tripoles had not been observed in geophysical flows, but these authors offered that "it is not unlikely that oceanic examples will be found in the near future." Shortly after, an observation was made in images taken by the NOAA 11 satellite of what appears to be a tripolar structure. The observation corresponds to a vortex of about $50-70 \mathrm{~km}$ in diameter, which lived for almost a year in the Bay of Biscay. ${ }^{31}$ This oceanic vortex, named F90, exhibited tripolar structure for only a few days, however. Therefore, it is not a definitive observation of an oceanic tripolar vortex.

Numerical evidence of a vortex tripole was first seen by Legras et al., ${ }^{32}$ who observed its spontaneous emergence in two-dimensional, forced, homogeneous turbulence. More detailed simulations showing flows evolving to tripoles were performed by Carton et al., ${ }^{33}$ who used spectral methods to study perturbed, linearly unstable axisymmetric vortices. 

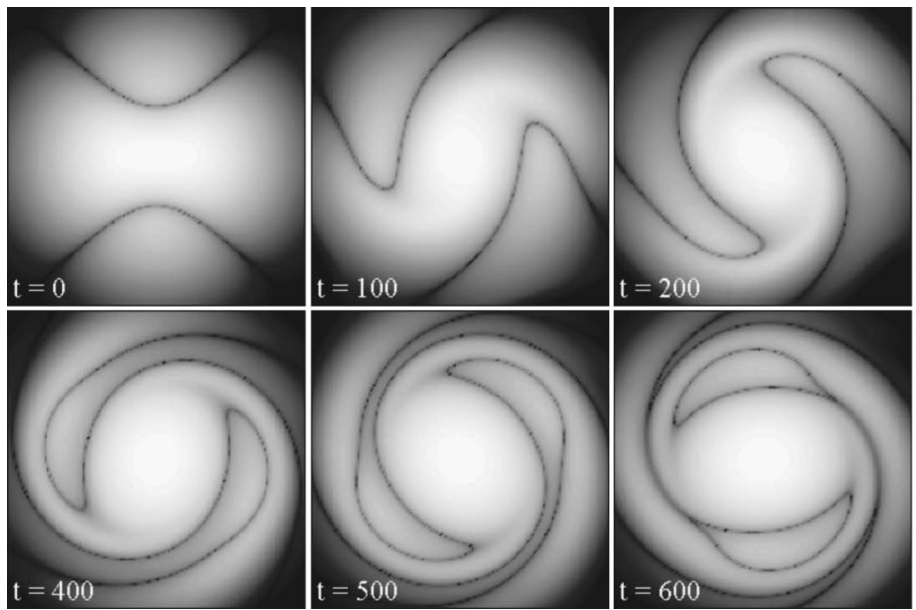

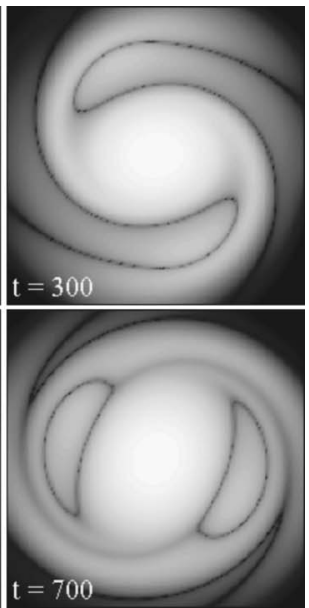

FIG. 2. Same case as Fig. 1; plot of the logarithm of $|\omega|$, emphasizing the level zero of vorticity. Black in the gray scale is saturated at level $10^{-6}$ (enhanced online).
They proposed as the mechanism for generation of tripoles the growth and saturation of instabilities. In turn, more detailed experiments were presented by van Heijst et al. $;^{34}$ these show the generation of compact tripoles from stirringinduced vortices, and the photographs provided in their paper are quite striking. Laboratory tripoles are usually formed in situations of rotating flow, with initial conditions consisting of isolated monopoles. An isolated or shielded vortex consists of a core of vorticity surrounded by a ring of opposite vorticity, with zero total circulation. These structures may experience the growth of a wavenumber 2 perturbation, leading to a drastic change in topology (the formation of the two disconnected satellites from the outer ring of negative vorticity). The present work, in contrast, does not study shielded vortices (which are unstable), but stable Gaussian monopoles, with a finite amplitude perturbation (which destabilizes them).

\section{DETAILED NUMERICAL STUDY}

\section{A. General characteristics of the flow evolution}

The numerical experiments in the present paper treat nonlinearly perturbed vortices with a quadrupolar perturbation which is often large. As shown in the first frame of Fig. 1 , the initial condition has regions of negative vorticity, which occupy two disconnected inclusions on opposite sides of the core of positive vorticity. One could in fact say that the initial condition is already a sort of tripole; it is, however, far from steady state. The tripole vortex obtained at later times (last frame of Fig. 1) is a quasisteady flow structure. During the flow evolution, perhaps similar to the case of shielded monopoles, there is a change in the flow topology which in this case can be described by the "pinching" of the contour level of zero vorticity, as seen in Fig. 2 to happen between $t=500$ and $t=600$.

Figure 2 presents a useful way to visualize the flow; it consists of gray-scale contour levels of the logarithm of vorticity. The absolute value of the vorticity is used, to avoid problems with the logarithm of a negative value, and the effect is one of emphasizing the region where the vorticity changes sign. This boundary between positive and negative vorticity winds up around the base vortex, and at some time pinches, forming the isolated satellites of negative vorticity that characterize a tripole. After this transient, during which the vorticity reorganizes into a quasisteady tripole, the structure decays slowly and sometimes exhibits oscillations.

In contrast to the situation depicted in Fig. 2, if the initial amplitude of the perturbation is (relatively) small, the boundary between positive and negative vorticity lies farther away from the core. Subsequently, as it winds up, it folds flat onto itself, without pinching to enclose a region of negative vorticity. This is the situation shown in Fig. 3, which corre-
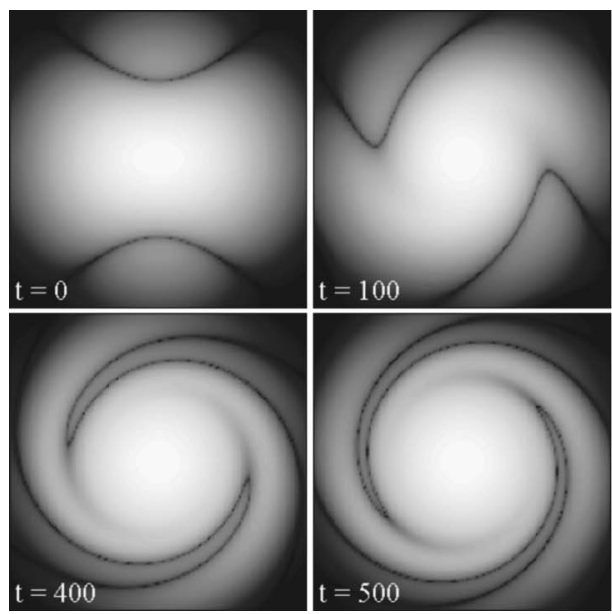
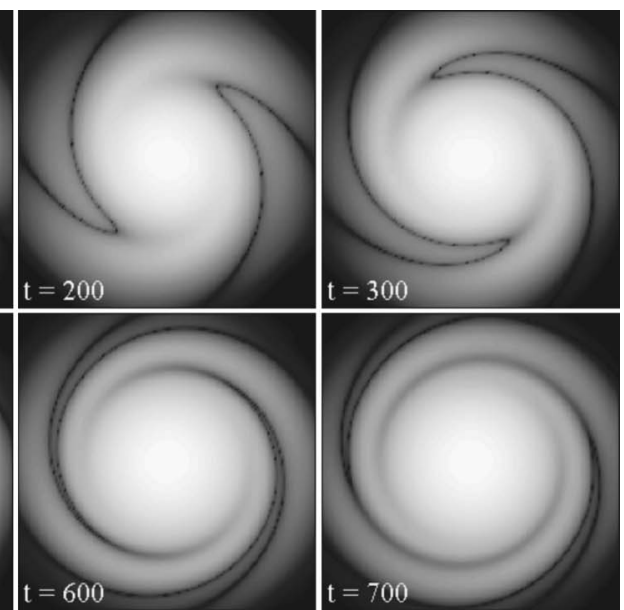

FIG. 3. Plot of the logarithm of $|\omega|$, emphasizing the level zero of vorticity; $\operatorname{Re}=10^{4}, \quad \delta=0.10 \quad$ (enhanced online). 




FIG. 4. Perturbation vorticity normalized by $\omega_{\max }(0) ; \operatorname{Re}=10^{4}, \delta=0.25$. Gray is zero, black is negative, white is positive (enhanced online).

sponds to $\delta=0.10$, for which the flow is seen to relax towards axisymmetry.

A further insight into the evolution of the flow for large and small amplitude initial perturbations is obtained from visualizing the perturbation itself. By "perturbation" at times $t>0$, we mean the field obtained from subtracting from the total vorticity the Lamb-Oseen solution. This is shown in Figs. 4 and 5 for the cases discussed above, i.e., $\delta=0.25$ and $\delta=0.10$ with $\operatorname{Re}=10^{4}$. For the larger amplitude, the perturbation vorticity starts winding up, but only the positive part forms a spiral structure. The negative perturbation remains as two separate inclusions, which come to form the tripole satellites. The spirals of positive perturbation become homogenized, and commence to merge towards the core, leaving weak filamentary debris that surrounds the whole structure. For the smaller amplitude initial perturbation, the same commences to happen, but the negative inclusions become squeezed in between the positive spirals. They become sheared and stretch around the core, and thus become homogenized. Of course, it is not entirely correct to speak of "perturbation vorticity" as we have, by just subtracting a spreading Gaussian from the total vorticity field, because the axisymmetric base flow and the nonaxisymmetric component are coupled; i.e., the flow is nonlinear for moderate ampli- tudes (as will be discussed). However, it is a useful means of visualizing the evolution of the flow and of characterizing the decay of the vortex structure.

A very interesting feature stands out in these plots of the perturbation. Notice in the last frames of Figs. 4 and 5 that a darker small region remains at the center of the vortex, indicating that the positive perturbation does not completely mix there. It has been found that the time scale of the sheardiffusion mechanism is slower at the center of a smooth vortex, ${ }^{35}$ where the gradient of angular velocity vanishes (and thus, there is no differential rotation). Instead of varying with Reynolds to the power $\frac{1}{3}$, the shear-diffusion time scale is order $\mathrm{Re}^{1 / 2}$ at the center of a vortex. We conjecture that this defect in shear-diffusion is evidenced in the darker center of the perturbation plots. In the case with the larger perturbation (Fig. 4), one can see that the center defect stands in the way of the final merging of the positive perturbation, which in turn contributes to retain an elliptical core, another characteristic feature of tripolar vortices.

\section{B. Parameter study}

We now consider situations in which the perturbation amplitude lies between the two cases discussed above, with

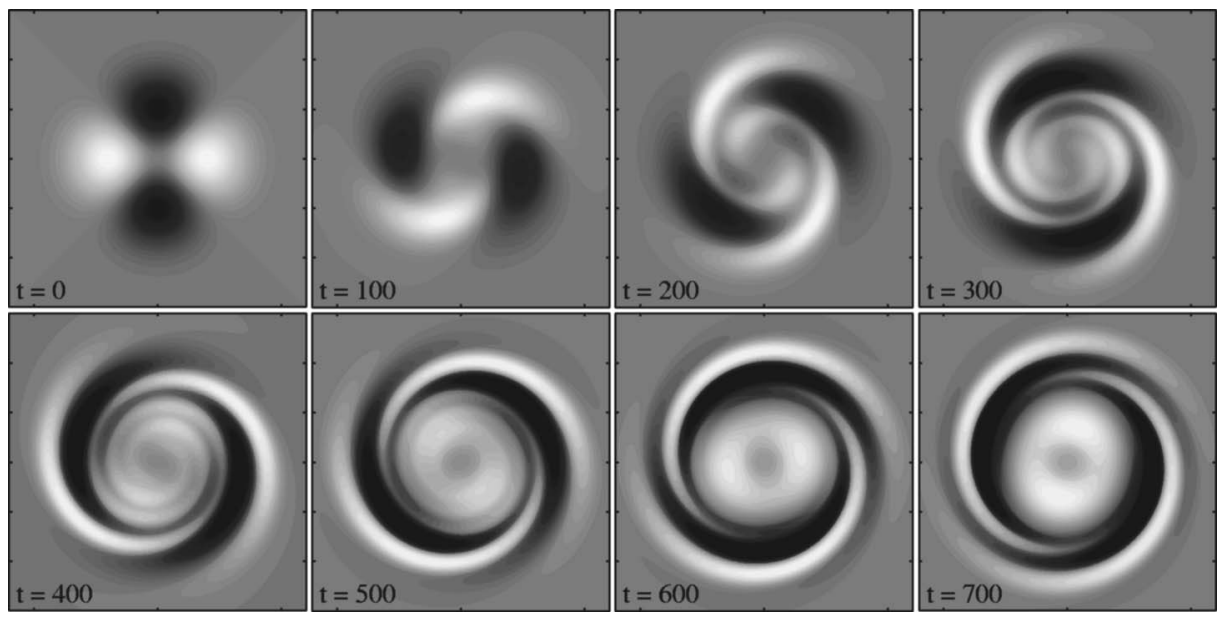

FIG. 5. Perturbation vorticity normalized by $\omega_{\max }(0) ; \operatorname{Re}=10^{4}, \quad \delta=0.10$. Gray is zero, black is negative, white is positive (enhanced online). 


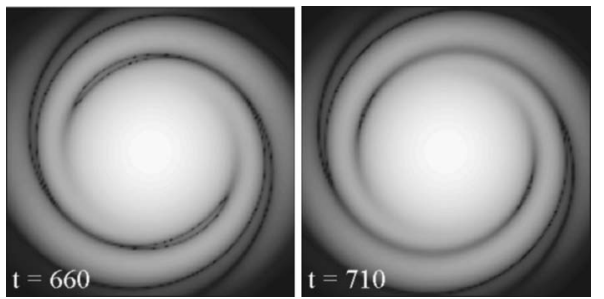

FIG. 6. Plot of the logarithm of $|\omega|$, emphasizing the level zero of vorticity; $\operatorname{Re}=10^{4}, \delta=0.125$.

the same Reynolds number. In these cases, by means of plotting the logarithm of vorticity as before, we observe that the zero-contour level of vorticity folds and pinches leaving a small satellite of negative vorticity, which decays in a short time. The size of these satellites increases with the value $\delta$, and thus they live longer for larger initial perturbations.

Figure 6 corresponds to a calculation with $\operatorname{Re}=10^{4}$ and $\delta=0.125$. The frames show the times when the zero-contour of vorticity pinches, and later when the narrow satellites that were formed diffuse away. Here we encounter a stumbling block: Is this a tripole? Structurally, it could be considered a tripole with extremely weak satellites (see Fig. 7). These very weak satellites disappear almost immediately, and the flow proceeds to become axisymmetric. The conundrum presents itself because we would like to determine a threshold amplitude for the emergence of the tripole, and moreover determine its relationship with Reynolds number. One could, for example, consider the case in Figs. 6 and 7 to be just barely above that threshold, as for larger values of $\delta$ all cases will develop a tripolar structure with larger and larger satellites.

In the first stage of this numerical study, 37 different simulations were carried out as part of a parametric study. Ten simulations were performed for a Reynolds number of $10^{4}$, with different amplitudes of perturbation between 0.1 and 0.25 . Eleven simulations were carried out for $\mathrm{Re}=3$ $\times 10^{3}$, with $\delta=0.1-0.3$, and sixteen for $\operatorname{Re}=10^{3}$ with $\delta=0.075-0.35$. That there is in fact a "threshold" amplitude separating two sorts of temporal behaviors is illustrated by the plot in Fig. 8. Each marker in the plot represents one simulation, parametrized by the initial amplitude of the perturbation $(\delta)$ and Re. The quantity on the plot's ordinate is the ratio of minimum to maximum vorticity in the flow (in absolute value). This ratio quantifies the importance of the negative inclusions with respect to the total structure, and thus it is a measure of the nonaxisymmetric state; the ratio

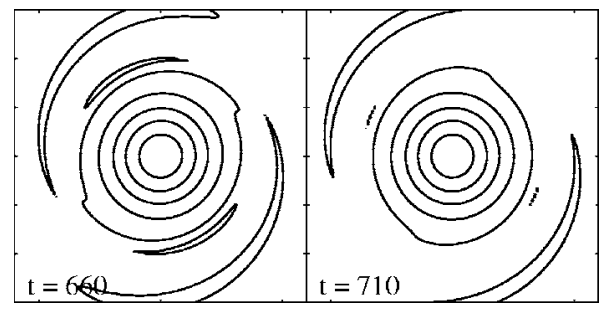

FIG. 7. $\operatorname{Re}=10^{4}, \delta=0.125$; vorticity normalized by $\omega_{\max }(0)$, contour levels at $\left[0.8,0.6,0.4,0.2,0.04,-1 \times 10^{-4}\right]$.



FIG. 8. Ratio of minimum to maximum vorticity $\left|\omega_{\min } / \omega_{\max }\right|$, at $t=600$, vs initial amplitude of the perturbation $\delta$ for the 37 simulations of the parameter study (each marker represents one simulation).

tends to zero over time, indicating that the negative satellites become mixed and decay. Rossi et al. ${ }^{9}$ plotted this quantity with respect to the viscous time, for three simulations with $\delta=0.25$ and different Reynolds numbers (their Fig. 9); they conclude that the tripolar structure decays by diffusion. On the abscissa of Fig. 8 is the value of $\delta$, and the runs with same Reynolds number have been joined by a dotted line. The quantity $\left|\omega_{\min } / \omega_{\max }\right|$ is shown for a time slice $t=600$ that corresponds to approximately four turn-over times, which is generally enough time for the initial transient when the flow reorganizes. It can be seen that there is a sharp decay of the negative inclusions at this time for a given value of $\delta$ that is smaller for larger Reynolds number.

To illustrate how the quantity $\left|\omega_{\min } / \omega_{\max }\right|$ varies in time, it is plotted in Fig. 9 for ten different simulations with $\operatorname{Re}=3 \times 10^{3}$, at different times. It can be seen that a sharp drop occurs in this diagnostic at a given time that varies with $\delta$. This is associated with the mixing of the negative vorticity inclusions, and thus when a tripole is present the sharp drop does not occur.

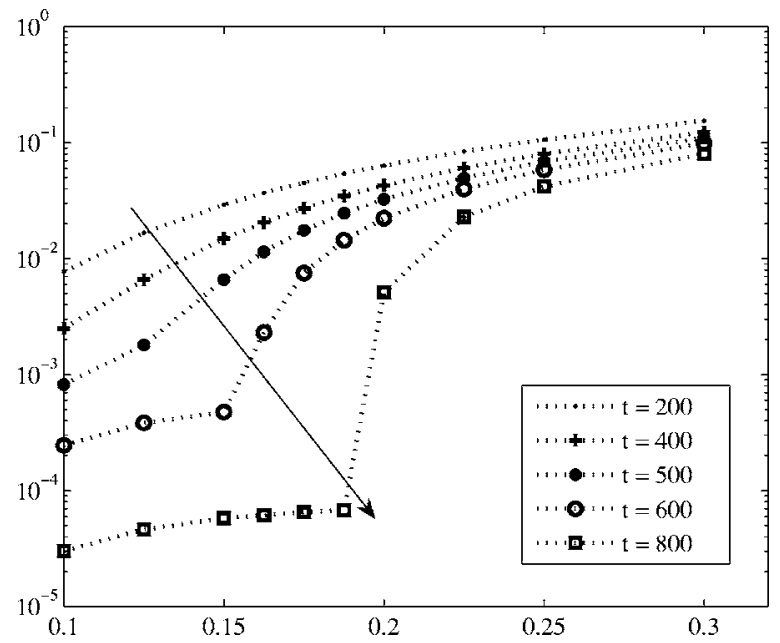

FIG. 9. Ratio of minimum to maximum vorticity $\left|\omega_{\min } / \omega_{\max }\right|$ vs initial amplitude of the perturbation $\delta$ for ten simulations with $\operatorname{Re}=3 \times 10^{3}$, at different times (the arrow indicates increasing time). 


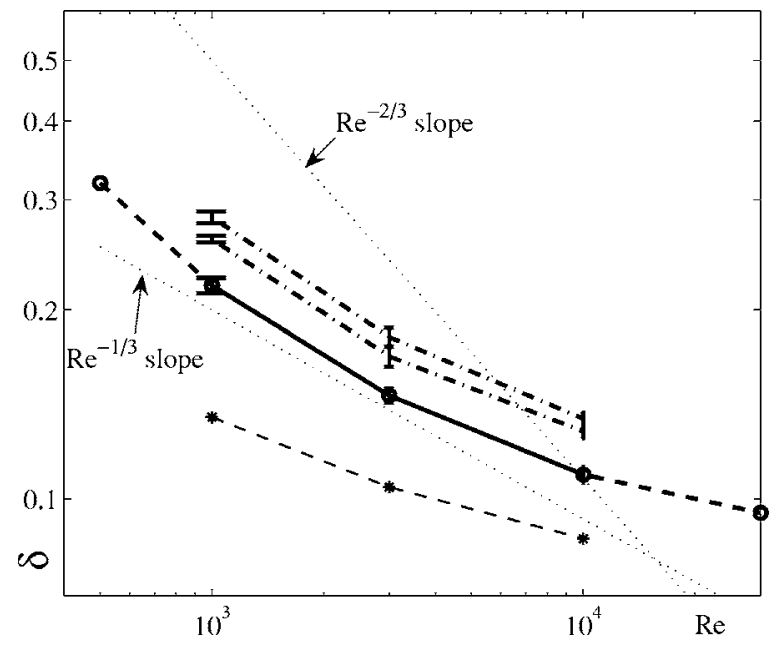

FIG. 10. Threshold amplitude of the initial perturbation $\delta$ vs Reynolds number: for pinching of zero-contour (solid line), or for half-turn or full-turn satellite survival (dash-dotted lines above).

The above discussion exposes the possibility of a bifurcation between the tripole and monopole states, as assumed by Rossi et $a l .^{9}$ It does not, however, aid us in determining the threshold value of the initial perturbation separating the two asymptotic solutions.

Let us for the moment consider the "pinching" of the zero contour of vorticity to indicate the emergence of a tripole, even though it may be very short lived. With this choice, we can find a "critical" or threshold amplitude of the (initial) perturbation above which the tripole forms, for each of the three values of Reynolds number used (so far). This is plotted in Fig. 10 as the three values joined by a solid bold line (disregard for the moment the dashed extensions to this line). Admittedly, this is a rather arbitrary definition of the threshold, but in the absence of a more categorical or better definition, the results in Fig. 10 seem to indicate an approximate $\mathrm{Re}^{-1 / 3}$ relationship for the critical amplitude (in the present range of Reynolds number). With a different choice or definition of what constitutes the threshold, perhaps another Reynolds number dependence might be found.

Let us now consider that such weak tripoles for which the satellites are very short lived should be discarded. One could decide, for example, that a tripole must exist for at least half a turnover time. With this new criterion, we find again the threshold initial perturbation for each Reynolds number, above which the satellites survive at least half a turn. That is, the time between the pinching of the zero contour of vorticity and the disappearance of the satellites of negative vorticity must be at least $\tau / 2$ with $\tau=4 \pi / \omega_{\max }$ (we consistently used $\omega_{\max }$ at $\left.t=0\right)$. Plotting the result of this exercise in Fig. 10 as the dash-dotted line just above the solid line, one finds once again a $\mathrm{Re}^{-1 / 3}$ relationship (approximately) for the critical amplitude. If, furthermore, one defines the threshold such that the satellites survive one full turn-over period, then the upper dash-dotted line is obtained, also displaying a $\mathrm{Re}^{-1 / 3}$ relationship. (In Fig. 10, the error bars show how closely two consecutive runs are, in their respective values of $\delta$, between which the chosen threshold lies.)

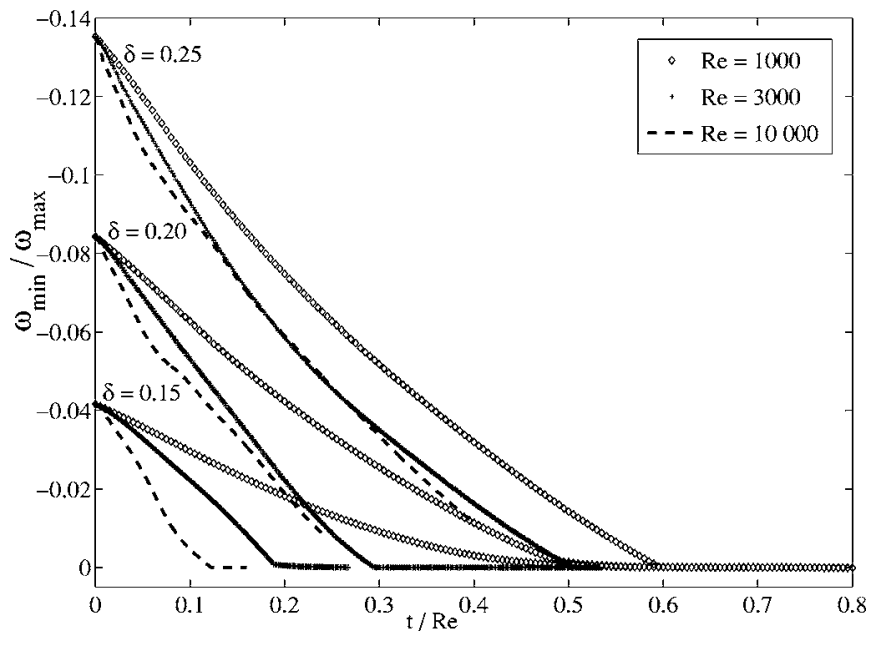

FIG. 11. Decay of the tripolar structure: ratio of minimum to maximum vorticity vs viscous time, for different values of $\operatorname{Re}$ and $\delta$.

The parameter study presented so far-Fig. 10 in particular-was partially motivated by the study of Le Dizès, ${ }^{36}$ in addition to Rossi et al., ${ }^{9}$ who proposed that there should be a critical value of $\delta$ separating tripoles from monopoles. Le Dizès utilized asymptotic methods to analyze slightly nonaxisymmetric vortices at high Reynolds number. In Le Dizès, the perturbation parameter measures the size $\epsilon$ of the nonaxisymmetric disturbance at the vortex core, once the asymptotic solution is established. Thus, it is not linearly related to our amplitude parameter $\delta$. This can be seen in Fig. 10 , where we also show the corresponding critical $\epsilon$ as a function of Reynolds number, computed using the methods of Sec. IV G below. (We calculated $\epsilon$ using the amplitude of the $m=2$ mode; the asterisks on the plot of Fig. 10 show the value of $\epsilon$ multiplied by a constant to fit in the same axis.) As a result of his analysis, Le Dizès he conjectured that the threshold amplitude for the existence of nonaxisymmetric states should decrease with increasing Reynolds number. That is clearly in agreement with the present numerical study. However, the results discussed above also seem to indicate a $\mathrm{Re}^{-1 / 3}$ relation, in the given range of $\mathrm{Re}$, which was not predicted by the asymptotics. Instead, the work of Le Dizès predicts a $\mathrm{Re}^{-2 / 3}$ relationship for the critical amplitude of the nonaxisymmetric component $(\mathrm{a}-2 / 3$ slope is drawn in Fig. 10 for comparison). He directly compared his asymptotic results with the numerical solutions found by Rossi et al., and argued that they correspond to the same solutions. Indeed, good agreement was shown not only in the co-rotating streamlines but also in the value of the angular frequency.

Perhaps the reason for the disagreement with the prediction of Le Dizès is that he based his study on an equation which is obtained at leading order. That is, higher-order nonlinear terms were neglected in the governing equation, representing a radial analog of the critical layer equation.

Nevertheless, we attempt to characterize the relationship between critical amplitude of the initial nonaxisymmetric component resulting in a tripole vortex, and Reynolds number. To verify whether the critical amplitude of the initial 

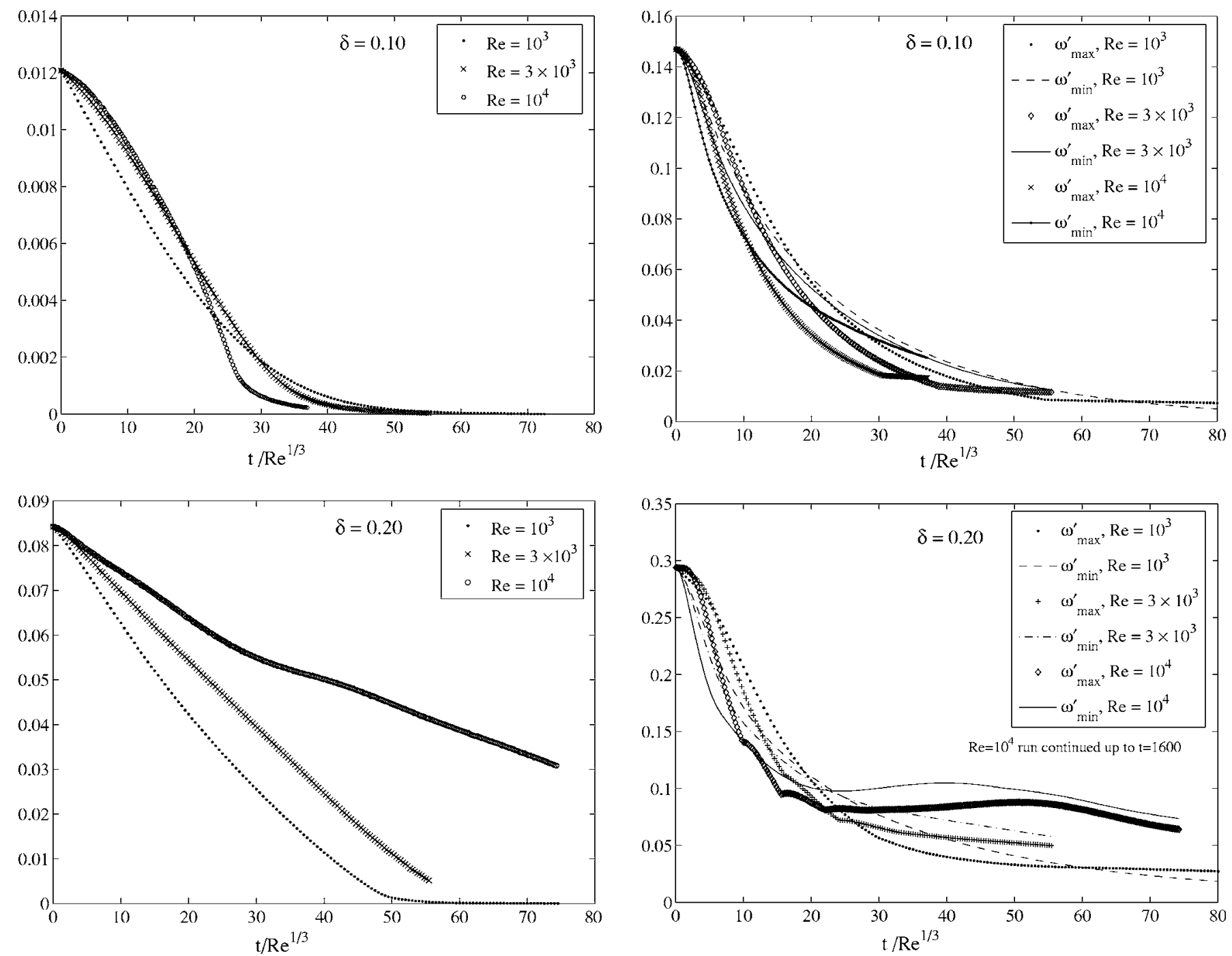

FIG. 12. Decay of the tripolar structure: ratio of minimum to maximum vorticity $\left|\omega_{\min } / \omega_{\max }\right|$, vs shear-diffusion time scale, for different values of Re and $\delta$.

perturbation follows a power law relationship with Reynolds number, new series of simulations were performed for $\operatorname{Re}=500$ and $\operatorname{Re}=3 \times 10^{4}$. We encounter some computational difficulty in the large or small Reynolds number range, due to increase of the required memory and CPU time. When Re is smaller, the vortex method with core spreading requires more frequent spatial adaption (to control core sizes), and also the vorticity support grows considerably due to viscous spreading. This leads to increased problem size and thus CPU time and memory requirements. On the other hand, when the Reynolds number is very large, the resolution must be increased, which also leads to increased problem sizes. For this reason, our capacity to extend the curve in Fig. 10 is limited (more effort can still be made to improve code efficiency, however). The new series with $\operatorname{Re}=3 \times 10^{4}$ spanned $\delta=0.09$ to 0.2 (eight simulations), and the new series with $\operatorname{Re}=500$ spanned $\delta=0.2$ to 0.375 (nine simulations). The threshold values for the formation of a closed zero-contour of vorticity were found and are plotted in Fig. 10, corresponding to the extensions with dashed line.

FIG. 13. Decay of the tripolar structure: negative and positive perturbation vorticity amplitude (max-norm) vs shear-diffusion time scale, for different values of $\operatorname{Re}$ and $\delta ; t_{o}=0$.

It is apparent that one cannot put forward a power-law relationship between Reynolds number and the threshold amplitude of the initial perturbation that leads to emergence of the tripole. The trend in Fig. 10 also indicates that there might be an inviscid limit, a critical value of $\delta$ above which the flow always organizes into a tripole in the large Reynolds number limit. This is intriguing and deserves further research.

\section{Time scale of relaxation}

Next, we consider the time-decay of the vortical structure. Figure 11 shows the evolution of the ratio between minimum and maximum vorticity, the minimum vorticity indicating the strength of the negative inclusions. Three different values of the initial perturbation amplitude and three different Reynolds numbers are shown; the decay is plotted with respect to $t / \mathrm{Re}$, the viscous time scale.

Rossi et al. ${ }^{9}$ considered the decay of the tripole structure with regards to this quantity; in Fig. 9 of their paper, they show only the case $\delta=0.25$ for three Reynolds numbers: $10^{3}$, 




$\varepsilon=0$.

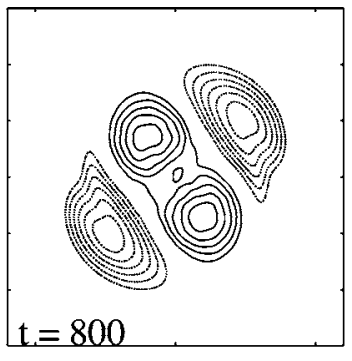

$\varepsilon=0.01$

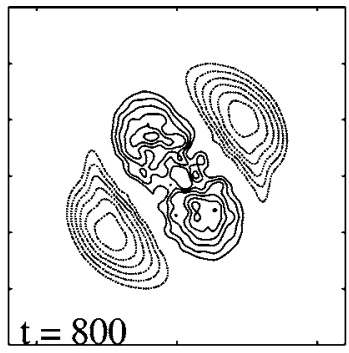

$\varepsilon=0.05$



$\varepsilon=0.1$

FIG. 14. Initial conditions for tripole runs with perturbed output case $\operatorname{Re}=10^{4}, \delta=0.25$. Contour levels $\pm[0.04: 0.02: 0.32]$ (higher contours not present due to decay of perturbation vorticity from $t=0$ ).

$5 \times 10^{3}$, and $10^{4}$. They conclude that the structure is decaying on the viscous time scale, as the evolution at various Re seems to scale well with $t /$ Re. We note, however, that by plotting the decay in the same manner for the smaller amplitude perturbations, the scaling no longer applies well (see Fig. 11). In fact, even for the larger $\delta=0.25$, our results do not exhibit a clear scaling with $\mathrm{Re}^{-1}$. As shown graphically and discussed in Ref. 23, our results disagree with those of Rossi et al. for the lower $\operatorname{Re}=10^{3}$, and we attribute this to the increased numerical diffusion introduced by the frequent splitting events required in Rossi's method. In consequence, our results exhibit a retardation in the decay for $\operatorname{Re}=10^{3}$, in comparison with Ref. 9.

Furthermore, Rossi et al. acknowledge that their "greatest frustration is that [they] do not observe rapid sheardiffusion mixing in the tripolar attractor." They speculate on a lack of numerical resolution hindering the observation of the mechanism, and also point out that the lack of an analytic solution for a tripole makes measurement of the relaxation problematic. We now address this issue of shear-diffusion in the evolution of this flow.

First, consider simply plotting the same quantity of Fig. 11 , but with respect to the shear-diffusion time scale: $t / \mathrm{Re}^{1 / 3}$. For small amplitude perturbations, one finds that this scaling does collapse rather well the evolution for different Reynolds numbers [see Fig. 12(a)]. As the amplitude of the perturbation is increased, however, the decay curves for the different values of Re start to grow apart and the scaling no longer works well [Fig. 12(b)]. This result indicates that sheardiffusion could be active in the attenuation of the small perturbations but not the large ones. This should not be surprising. Shear-diffusion is a linear mechanism, and as discussed earlier vorticity perturbations can only be considered passive if they are very small. When the perturbation is large, nonlinear effects are important or even dominant, and there is no reason to expect a $\mathrm{Re}^{1 / 3}$ time scale (so perhaps the frustration of Rossi et al. was not merited). At large $\delta$, all that can be observed is a viscous decay of the tripolar structure, as shown in Fig. 11. The reorganization of the vorticity leading to emergence of the tripole is not effected by shear-diffusion alone; rather, it is produced by a combination of viscosity and nonlinear effects in an interplay that may possibly include some dynamical instability.

Now, consider the decay of the perturbation vorticity only. Plotting the maximum (positive) and minimum (nega- tive) perturbation field values as they evolve, it can be seen again that the $\mathrm{Re}^{1 / 3}$ time scaling appears to apply well only for the small perturbations. As shown in Fig. 13, once the perturbation amplitude (max-norm) is large, the behavior becomes more complicated. Indeed, for large Re and large $\delta$, the perturbation visibly oscillates during the selforganization stage.

\section{Stability of the tripole structure}

To test the robustness of the tripole vortex, we performed the following experiments. The final state of the simulation to a time $t=800$ (five turn-over times) of the case with $\operatorname{Re}=10^{4}$ and $\delta=0.25$ is used as initial conditions. Before initiating a continuation run, the particle positions are perturbed randomly, as in a random walk with a step $\epsilon / 2$. Using the perturbed particles, a continuation run is performed up to $t=1600$. Three continuation runs were carried out, with $\epsilon=0.01,0.05,0.1$ (where the value 0.1 is comparable to $h$, the interparticle spacing). The initial conditions thus obtained are shown in Fig. 14, which includes plots of equal contour levels of the perturbation vorticity for the three randomly perturbed cases and the unperturbed one; i.e., Fig. 14(a) corresponds to the same field as that shown in the last frame of Fig. 4.

The evolution of the case with $\epsilon=0.01$ is practically indistinguishable from the unperturbed case, whereas both other cases also eventually relax back to the tripole solution. The perturbation vorticity at two different times is plotted in Fig. 15 for the case $\epsilon=0.1$ and for the unperturbed case. It can be seen that the difference in $\omega^{\prime}$ is very slight by $t=1600$. The flow self-organizes back to the unperturbed evolution, which is also evident by plotting the normalized perturbation max-min field values, shown in Fig. 16.

It is quite clear that the tripole is a very robust structure, once formed. We consider this to be evidence that the tripole is indeed an asymptotic solution. There remain important questions about what are the mechanisms involved in its formation, the understanding of which merit further research.

\section{E. Connection with mixing theory}

Similarly to the process of vortex merging, which occurs in several stages of different characteristic time scales, one can try to characterize the nonlinear relaxation studied here in terms of stages. In the beginning of the first stage, one can 


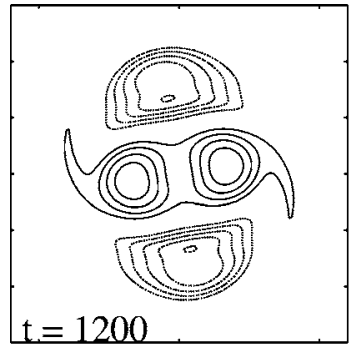

$\varepsilon=0$.

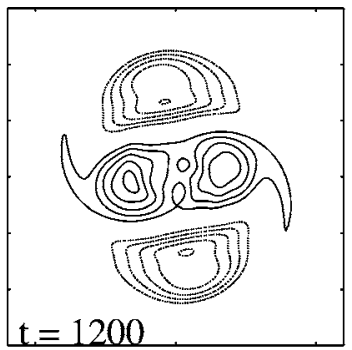

$\varepsilon=0.1$



$\varepsilon=0$.

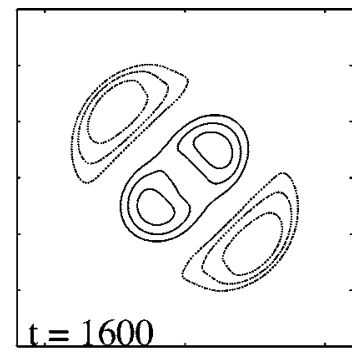

$\varepsilon=0.1$

FIG. 15. Perturbation vorticity at two times for continuation runs in the case $\operatorname{Re}=10^{4}, \delta=0.25$. Contour levels $\pm[0.04: 0.02: 0.32]$ (higher contours not present due to decay of perturbation vorticity from $t=0$ ).

conjecture that the process of mixing is active. When the amplitude of the perturbation is small, this is the only relevant stage, and shear-diffusion dominates at a $\mathrm{Re}^{1 / 3}$ time scale. However, with large perturbations, nonlinear effects become important. In particular, it seems that only the positive perturbation is responding to mixing processes when the amplitudes are large.

To support the claim that mixing is active in the first stage of the process, we look for evidence of the mixing time, that is, the characteristic time for the initiation of decay of the perturbations. This matter was discussed in detail by Meunier and Villermaux, ${ }^{37}$ who performed careful experiments of dye mixing in a Lamb-Oseen vortex. They were able to develop a nearly exact description for this problem, determining the evolution of the scalar concentration and an estimate of the mixing time. We use their result that the maximal concentration of scalar, observed at the center of a spiral branch, at radial distance $r$ from the center of the base vortex, evolves as [their Eq. (3.11)]

$$
c_{M}(r, t)=c_{0} \operatorname{erf}\left(\frac{1}{\sqrt{\tau}}\right)
$$

where $c_{M}$ is the maximal concentration, $c_{0}$ is the initial concentration, and

$$
\tau=\frac{D}{s_{0}^{2}} t\left(1+\frac{\Gamma^{2} t^{2}}{3 \pi^{2} r^{4}}\right) .
$$

In these equations, the symbols represent: $\Gamma$, the circulation of the base vortex; $r$, the radial location of the drop of scalar with respect to the center of the vortex; $s_{0}$, the initial size of the scalar drop; and $D$, the molecular diffusion rate. Noting that the scalar concentration in portions of a spirally deformed drop is constant until the mixing time, denoted $t_{s}$, they are able to obtain an estimate for this time (the concentration varies as an error function, the argument of which is order 1 until the mixing time, which gives the result). We reproduce here their Eq. (3.12):

$$
t_{s}(r)=\frac{r^{2}}{\Gamma}\left(\frac{3 \pi^{2}}{16}\right)^{1 / 3}\left(\frac{s_{0}}{r}\right)^{2 / 3}\left(\frac{\Gamma}{D}\right)^{1 / 3} .
$$

Note that the mixing time depends on $\mathrm{Pe}^{1 / 3}$, where $\mathrm{Pe}$ $=\Gamma / D$ is the Péclet number. After the mixing time, there should be a decay of the concentration scaling as $t^{-3 / 2}$, the limiting slope of the error function.
For the case with $\operatorname{Re}=10^{4}$ and $\delta=0.25$, for which the evolution of the perturbation amplitudes is depicted in Fig. 21 , we plot the expected scalar concentration evolution according to Eq. (3). The correct parameters for this case are $D \rightarrow \nu=10^{-4}, \Gamma=1.0$, and $r=2$, which is the radial location of the maxima of the perturbation initially, and $s_{0}=2.0$, which is the characteristic size of the initial perturbation peaks. Note that none of these parameters is adjustable, they are given by the initial flow conditions only. The initial maximal vorticity perturbation is 0.3673 when using $\delta=0.25$.

As shown in Fig. 17, the evolution of the positive part of the perturbation seems to capture very well the mixing time [the time at which the scalar concentration according to Eq. (3) starts to drop]. The negative perturbation, it seems, does not evolve according to mixing principles at any time. When performing the same analysis for the smaller value of $\delta=0.1$, however, the results are more difficult to interpret. This case is presented in Fig. 18, and one can see that the maximum of the perturbation (the positive maximum indicated by the diamond markers) starts to drop approximately at the mixing time. The diagnostic used, as in Fig. 17, is the maximum perturbation value in the whole field, whereas the mixing theory of Meunier and Villermaux refers to the concentration peak at the same radial location with respect to the center of the vortex. It can be seen when visualizing the

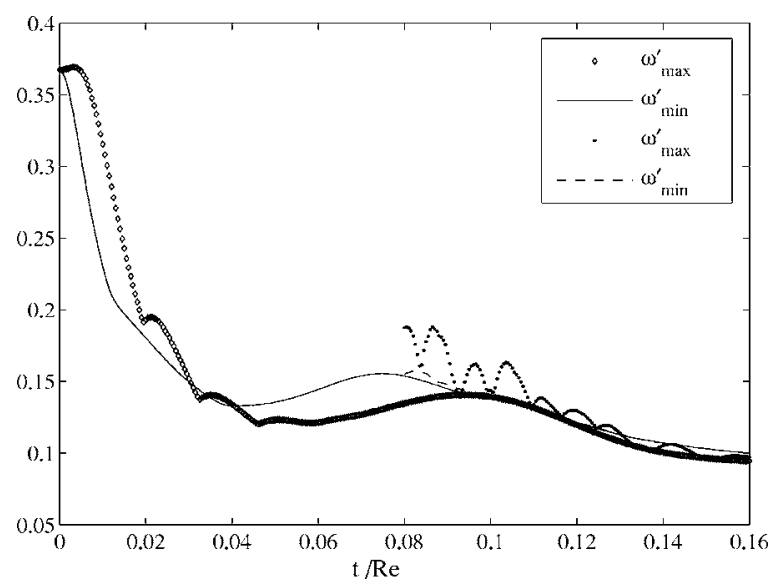

FIG. 16. Perturbation vorticity amplitude (max-norm) normalized by $\omega_{\max }(0)$ vs viscous time, for $\operatorname{Re}=10^{4}$ and $\delta=0.25$; continuation run up to $t=1600$ of perturbed case with $\epsilon=0.1$ on the same plot as unperturbed case (see text). 


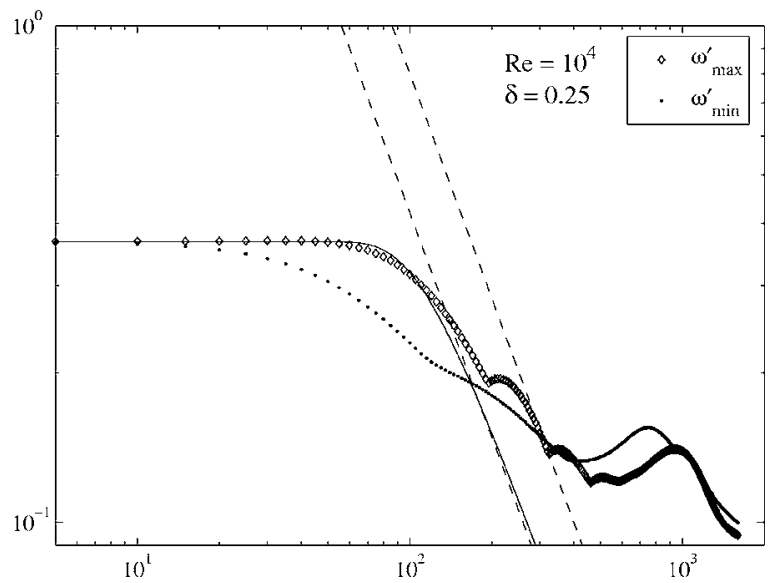

FIG. 17. Perturbation vorticity magnitude normalized by $\omega_{\max }(0)$ vs time on a $\log$-log plot; $\operatorname{Re}=10^{4}$ and $\delta=0.25$. Evolution of maximal concentration according to Eq. (3) in continuous line, and $t^{-3 / 2}$ slope in dashed line.

perturbation vorticity that the peak is on the spiral arms and is being expelled to larger radii (Fig. 5). In the previous case of $\delta=0.25$ this did not present a problem, because the perturbation maximum does remain at approximately the same radial location (this can be seen in Fig. 4). If we plot the maximum (and minimum) perturbation found on a circle of initial radius equal to 2 , which spreads at the rate of a LambOseen vortex, then the decay is considerably faster. This diagnostic is shown in Fig. 18 corresponding to the open circle and asterisk markers. The effect shown responds to the fact that the maximum of the perturbation is in fact being expelled to larger radius, as it occurs in the passive scalar case.

In brief, there is some evidence that the mechanism that is active in the most early stage of the relaxation is mixing, though it is inconclusive. It is quite remarkable, however, that the evolution of the positive part of the perturbation captures the mixing time so well. Once again, note that there are no adjustable parameters in Eq. (5). In any case, it is only the positive part of the perturbation that is susceptible to this mechanism, whereas the negative part decays more rapidly (earlier than the mixing time) in the initial stage. The visualization of the perturbation vorticity shows that the positive part is subject to spiral wind-up most intensely. The negative part, in contrast, appears to resist spiral wind-up, which may be attributed to nonlinear effects.

\section{F. Evolution stages and long-time evolution}

A series of continuation runs were carried out, to observe the long-time evolution of the tripole. Consider the case $\operatorname{Re}=3 \times 10^{3}$ and $\delta=0.25$. Figure 19 shows the logarithm of

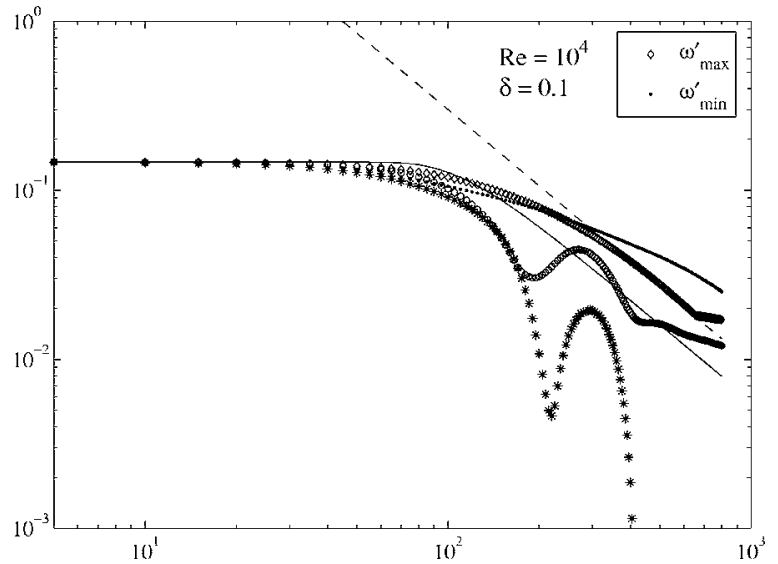

FIG. 18. Perturbation vorticity magnitude normalized by $\omega_{\max }(0)$ vs time on a $\log -\log$ plot; $\mathrm{Re}=10^{4}$ and $\delta=0.1$. Evolution of maximal concentration according to Eq. (3) in a continuous line, and $t^{-3 / 2}$ slope in dashed line. The additional curves (open circles and asterisks) correspond to $\omega^{\prime}$ measured on a spreading circle of initial radius $r=2$.

vorticity for the time when the zero contour pinches, the end of the first simulation $(t=800)$, and the time on a continuation run when the satellites are about to disappear. The negative satellites in this case survive for about six turn-over times. With $\operatorname{Re}=10^{4}$ and $\delta=0.25$, the zero vorticity contour pinches at $t=580$ and the negative inclusions survive for a very long time indeed. As seen in Fig. 20, the satellites are slowly getting smaller, but by $t=4000$ they still survive; this is about 25 turn-over times. They finally die out at $t=4600$. Indeed the tripole seems to be a robust, quasisteady structure.

Studying the case $\operatorname{Re}=10^{4}$ in the last frame of Fig. 13, which shows the decay of the perturbation amplitude, it is apparent that the relaxation of the flow consists of several stages. Consider Fig. 21, which shows the evolution in the viscous time of the amplitude of positive and negative perturbations, for a continuation run up to $t=3200$ of the case $\operatorname{Re}=10^{4}, \delta=0.25$. First, there is a rapid decline of the perturbation, followed by a nonlinear adjustment, and finally a very slow evolution. It has previously been asserted by other authors that this slow stage is simply viscous decay. Note that in the final stage depicted, the amplitude of the perturbations is between 0.05 and 0.1 (normalized by $\omega_{\max }$ at $t=0)$, so it is still not very small.

The intermediate stage in the relaxation process is one where oscillations of the perturbation suggest an interplay of dynamical effects. This is evident in particular in the large amplitude cases. During this stage, the flow reorganizes into the tripole, which is usually completely formed after between three and four turn-over periods (the time taken for the zero


FIG. 19. Plot of the logarithm of $|\omega|$, emphasizing the level zero of vorticity; $\operatorname{Re}=3 \times 10^{3}, \delta=0.25$. 

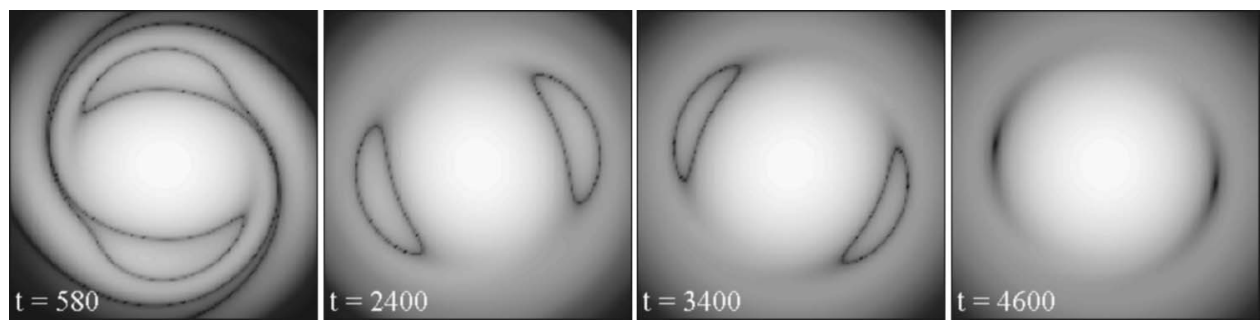

FIG. 20. Plot of the logarithm of $|\omega|$, emphasizing the level zero of vorticity; $\operatorname{Re}=10^{4}, \delta=0.25$.

contour of vorticity to pinch, enclosing the satellites). The physical mechanisms at play in this self-organization process are not well understood.

\section{G. Nonlinear evolution of the tripole}

We have mentioned that the reorganization of the tripole, and the decay of the nonaxisymmetric component, exhibit an oscillatory behavior. The oscillations are particularly apparent when looking at the decay of the perturbation for the higher Reynolds number and larger $\delta$ cases, as seen in Fig. 13 . To educe the oscillatory behavior, we look at the $L^{2}$-norm of the perturbation vorticity as it evolves in time. We have also performed simulations at larger Reynolds number, specifically $\operatorname{Re}=3 \times 10^{4}, 10^{5}$, with $\delta=0.2$. As seen in Fig. 22, the oscillations are damped more strongly with decreasing Reynolds number, suggesting viscous effects.

We have also acknowledged that there are limitations in using the perturbation vorticity, as defined here, to characterize the evolution, because there may be in fact transfer of energy to and from the axisymmetric component of the flow. Hence, to complete the analysis of the problem, we now look at the first few Fourier modes of the vorticity field.

Using the following definition of the modes, we calculate them for $n=0,2,4$ :

$$
\Phi_{n}=\frac{1}{2 \pi} \int_{0}^{2 \pi} \omega(r, \theta, t) e^{i n \theta} d \theta .
$$

We numerically obtain the modes from the vorticity field values on a sampling grid, and choose to look at these in particular for the case shown in Fig. 22 with $\mathrm{Re}=10^{5}$ and

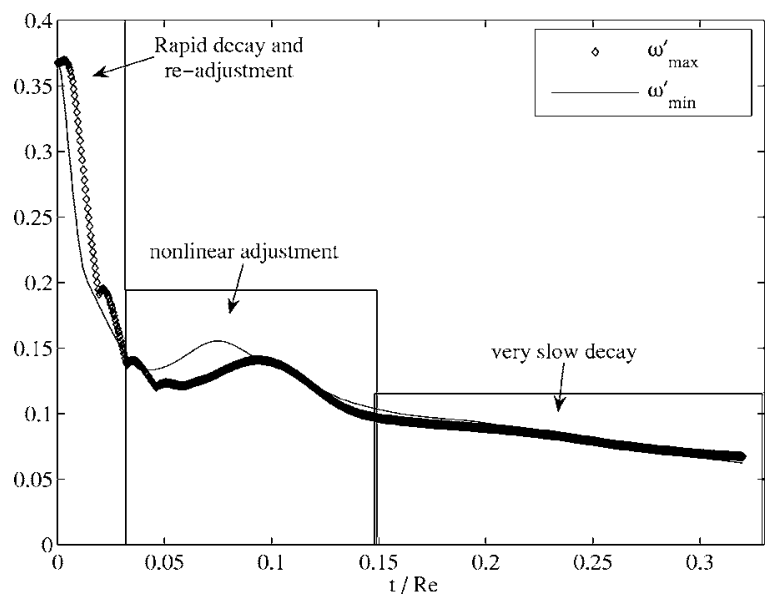

FIG. 21. Perturbation vorticity normalized by $\omega_{\max }(0)$ vs viscous time, for $\operatorname{Re}=10^{4}$ and $\delta=0.25$; continuation run up to $t=3200$. $\delta=0.2$, for three different times. The times chosen correspond as closely as possible to the first minimum of the plot, the first maximum after this, and the second minimum. These happen at times $t=600, t=1100$, and $t=1700$, respectively, as can be seen in the plot.

First, compare the radial distribution of the amplitude of the mode $\Phi_{0}$ for the three times, with a Gaussian distribution. This is shown in Fig. 23. Clearly, there are deviations from the Gaussian distribution. In the inner region, the amplitude of the zeroth mode is slightly larger than a Gaussian; in the tail, it is lesser than the Gaussian and it features a valley and local maximum. Most interestingly, the plot shows how this tail snakes up and down at different times. At $t=600$ (continuous line), the tail exhibits the greatest deviation from the Gaussian. This corresponds to the time in Fig. 22(c) when the $L^{2}$-norm of the perturbation vorticity reaches its first minimum. At $t=1100$ (dashed line), the distribution of $\Phi_{0}$ is closest to the Gaussian, but one still detects a small hump on the tail (around $r=5$ ). For the next time, i.e., $t=1700$ (dash-dotted line), $\Phi_{0}$ has again deviated from the Gaussian. This series of $\Phi_{0}(r)$ plots displays clearly that the axisymmetric mode of the vorticity is exchanging some energy with the nonaxisymmetric component, in an oscillatory fashion. We suggest that this is a manifestation of the nonlinear character of the evolution.

Figure 24 shows the amplitudes for the three modes $\Phi_{0}$, $\Phi_{2}, \Phi_{4}$, at the three times discussed in the previous paragraph. The plots for $\Phi_{2}$ show how this mode grows and shrinks during one oscillation, and in addition displays a radial rearranging. The $\Phi_{4}$ mode is mostly confined to the larger radii (around $r=4$ ), indicating perhaps that it captures the filaments around the structure.

The three modes were also calculated for the main cases discussed in the beginning sections of the paper, with $\operatorname{Re}=10^{4}$ and $\delta=0.25$ and $\delta=0.1$; recall that for the first case, the flow develops into a quasisteady tripole, whereas for the second case it evolves towards axisymmetry. For the larger $\delta$, we can see that the 2-mode decays to about half its amplitude between $t=100$ and $t=750$, but its radial distribution changes little, with the peak moving only slightly from $r \approx 2$ to $r \approx 2 / 5$ (see Figs. 25 and 26). There are several differences with the case with smaller $\delta=0.1$; first, the amplitude of the 2-mode was already much smaller at the early time, $t=100$ (in comparison with the previous case with larger $\delta$ ). Most notably, at the later time, $t=750$, the mode had experienced considerable radial rearrangement, with the peak now close to $r=4$. This is now a signature of the leftover spiral arms, as the satellites of negative vorticity have 

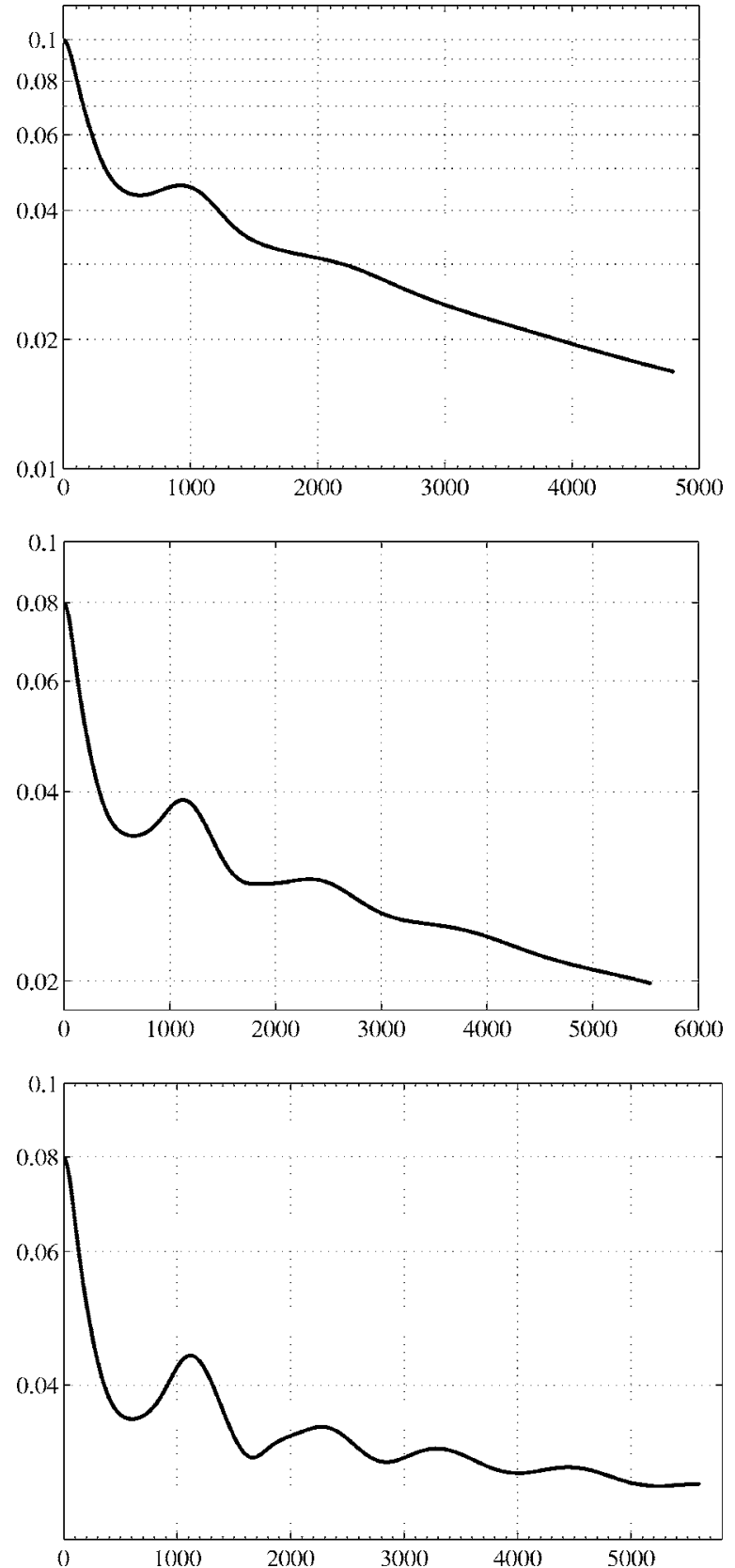

FIG. 22. Decay in time of the $L^{2}$-norm of the perturbation vorticity, for three simulations at large Reynolds number.

completely decayed. The maxima are expelled to larger radii, as would also be the case for shear-diffusion of passively small perturbations.

\section{CONCLUSIONS}

A two-dimensional vortical flow consisting of a LambOseen vortex with a quadrupolar perturbation of finite amplitude reorganizes into a quasisteady tripole for large enough perturbation. This paper presents a systematic numerical study of this flow situation, including a parametric study (in terms of Reynolds number and amplitude of the initial perturbation), and a detailed examination of the longtime relaxation. The parametric study was partly motivated by previous numerical results of Rossi et al., ${ }^{9}$ by which it

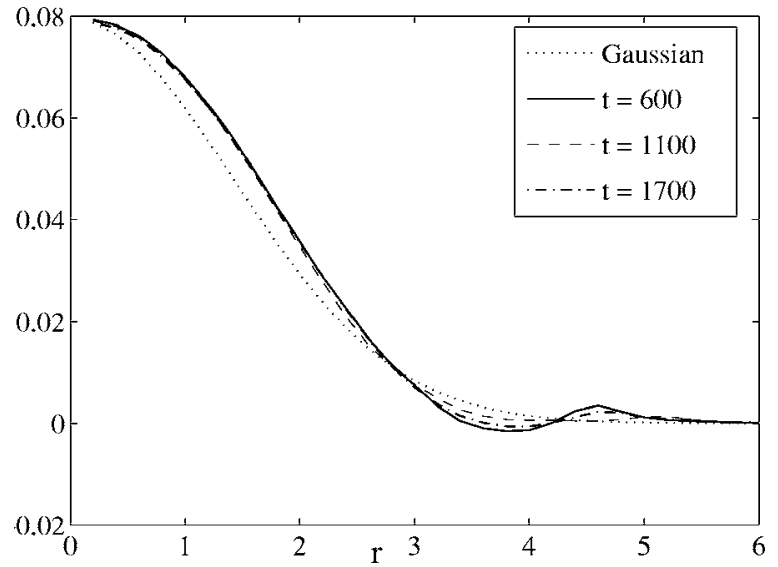

FIG. 23. Amplitude of the mode- $0, \sqrt{\left|\Phi_{0}\right|^{2}}$ at three different times, for the case $\operatorname{Re}=10^{5}$ and $\delta=0.2$.

was argued that a threshold perturbation amplitude might exist separating the domains of attraction of two asymptotic solutions: the monopole and the tripole. It was also partly motivated by asymptotic studies of Le Dizès, ${ }^{36}$ showing that slightly nonaxisymmetric vortices could survive without external forcing, and that the amplitude of the nonaxisymmetric correction should exhibit a $-2 / 3$ power law with respect to Reynolds number.

Analyzing the results of several simulations with each of three different Reynolds numbers, it was first observed that a critical or threshold amplitude of the initial perturbation leading to the emergence of the tripole behaved approximately as $\mathrm{Re}^{-1 / 3}$. Additional series of simulations with larger and smaller values of the Reynolds number indicated that the power law relationship only applies in a limited range of Re. As the Reynolds number is increased, the slope of the curve for critical amplitude decreases. This, in turn, suggests that there might be an inviscid limit, i.e., a critical value of the initial perturbation amplitude above which the flow always reorganizes into a tripole in the large Reynolds number limit. Thus, we find that the theoretical results of Le Dizès do not properly apply to the flow that we have studied. This is likely the result of considerable nonlinear effects which cannot be captured in the theory based on a leading order equation. The model of Le Dizès applies in the limit of small perturbations and large Reynolds number.

Detailed analysis of the simulation data was used to provide physical insight on the flow situation. A relaxation in the shear-diffusion time scale is apparent for cases when the amplitude is relatively small, but only a viscous time scale can be extracted from cases with large amplitude. This is explained by the fact that shear-diffusion is a linear mechanism, for passive scalars, and in the present flow nonlinear effects are dominant when the perturbation has large amplitude.

The very slow decay of the perturbation vorticity after the initial reorganization stage suggests the possibility of a nonlinear amplification mechanism. In fact, we have found that there is transfer of energy from the nonaxisymmetric modes to and from the axisymmetric mode of the vorticity.

The tripole proves to be a very robust structure, as 

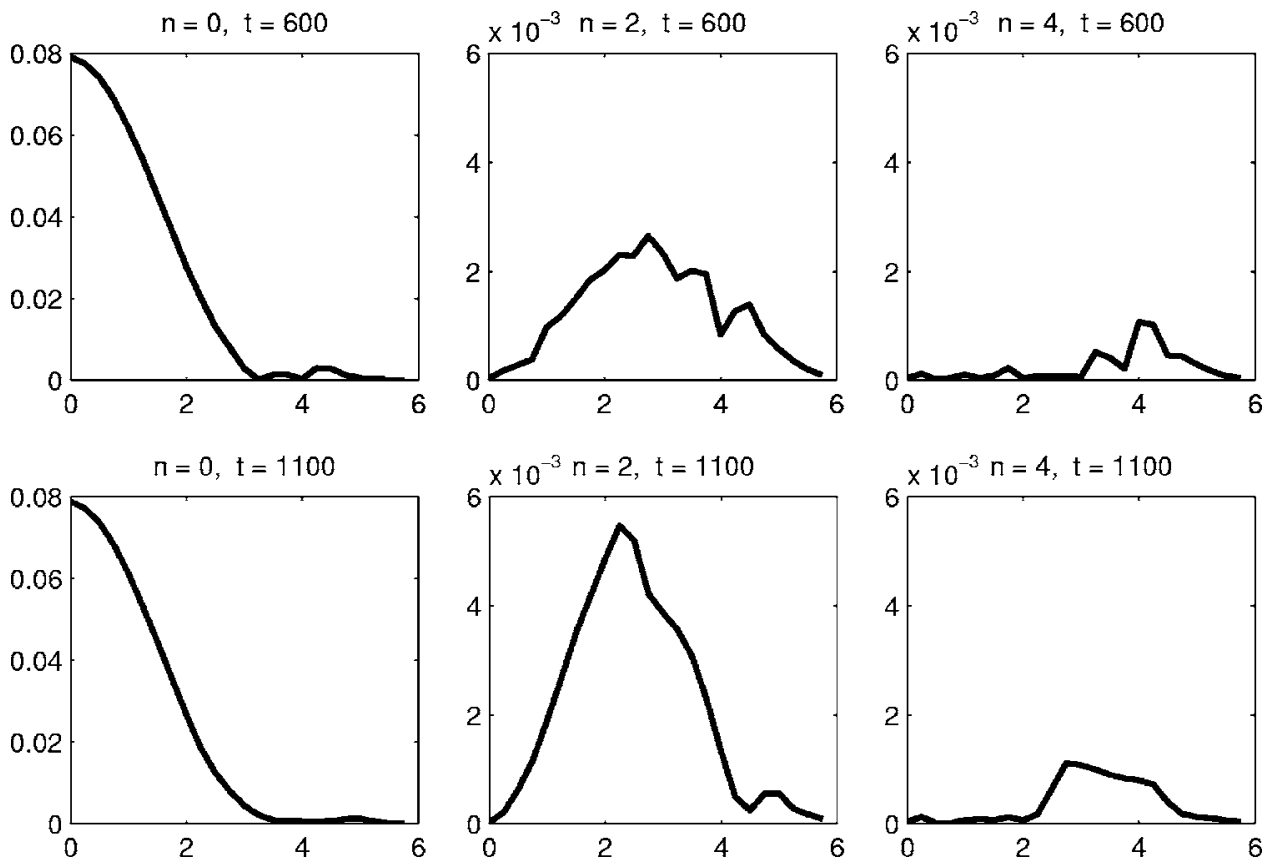

FIG. 24. Amplitude of three modes, i.e., $\Phi_{0}, \sqrt{\left|\Phi_{2}\right|^{2}}, \sqrt{\left|\Phi_{4}\right|^{2}}$ as a function of radius; same case and times as Fig. 23.
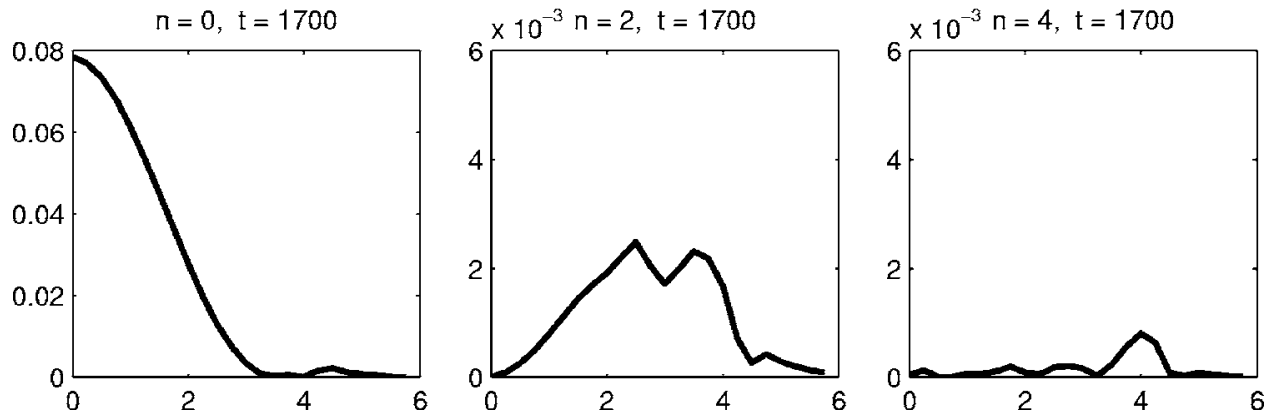

shown by experiments where a random perturbation was introduced to the vortex particles at an intermediate time. For perturbations of the particle locations as large as to be in the order of the interparticle spacing in the vortex method, the flow self-organizes back to the tripole solution. This result is

considered evidence that the tripole is indeed an asymptotic solution of the Navier-Stokes equations. The mechanisms involved in the tripole's formation, however, are not well understood, and warrant further investigation.

In summary, this work connects with the previous
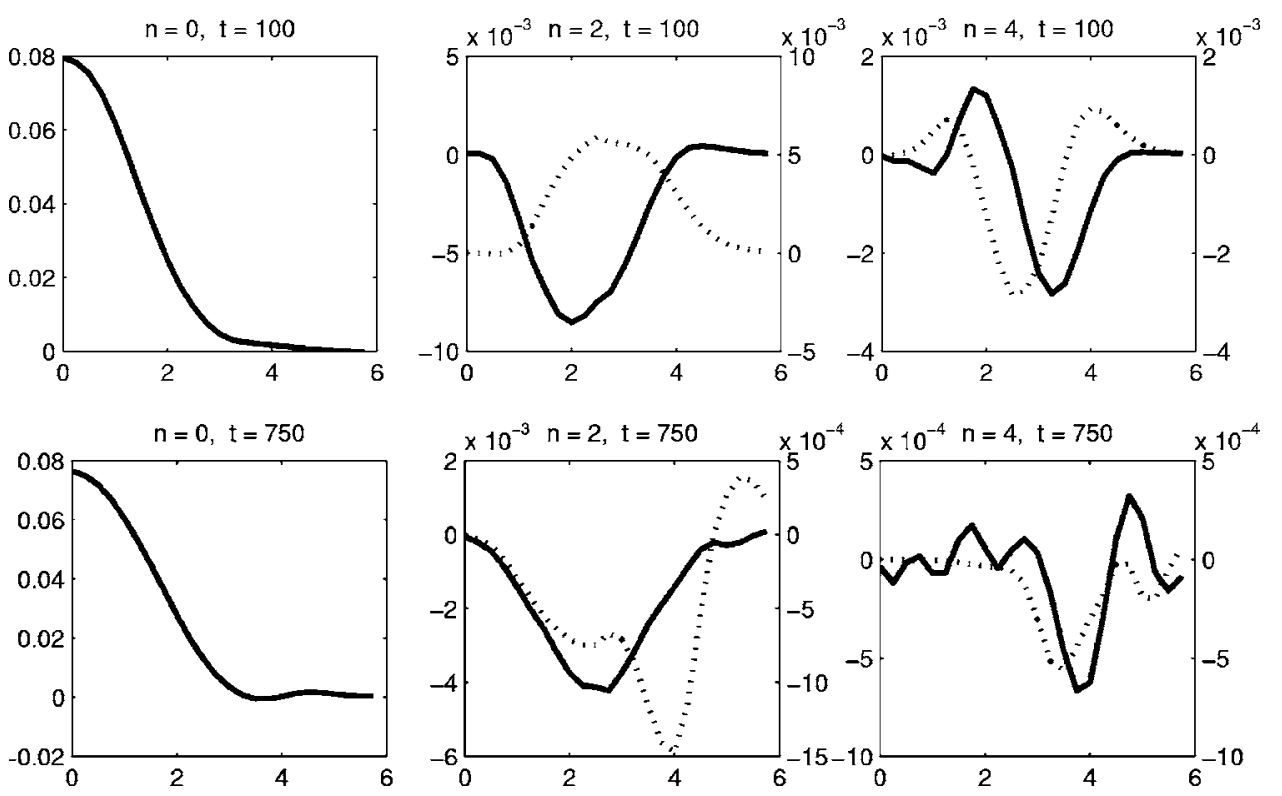

FIG. 25. For the three modes, i.e., $\Phi_{0}$, $\Phi_{2}, \Phi_{4}$, the solid line corresponds to the real part, and the dotted line corresponds to the imaginary part; case $\operatorname{Re}=10^{4}, \delta=0.25$, at the indicated times. (Note that the scale of the $y$ axis varies from plot to plot.) 

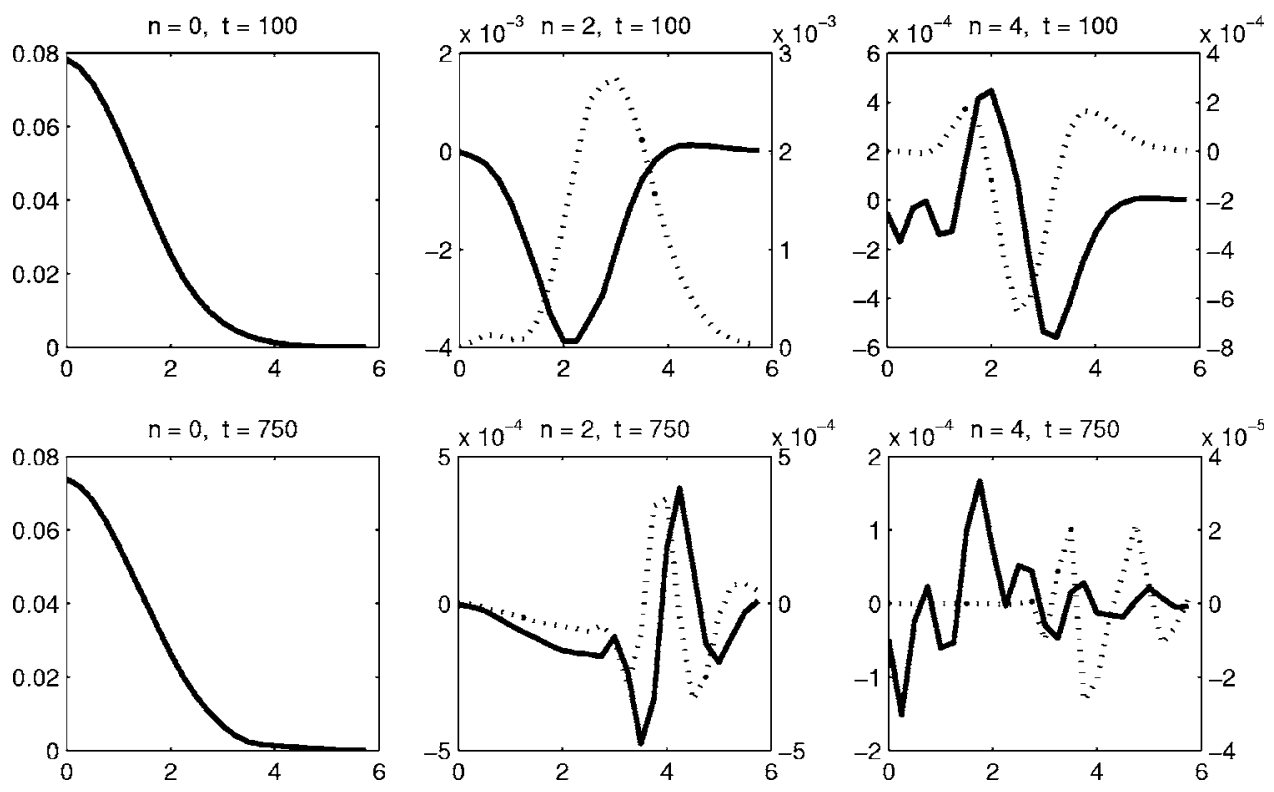

FIG. 26. For the three modes, i.e., $\Phi_{0}$, $\Phi_{2}, \Phi_{4}$, the solid line corresponds to the real part, and the dotted line corresponds to the imaginary part; case $\operatorname{Re}=10^{4}, \delta=0.01$, at the indicated times. (Note that the scale of the $y$ axis varies from plot to plot.)

literature ${ }^{9,36}$ by seeking to determine a threshold amplitude of the nonaxisymmetric component of the flow such that a tripolar vortex can survive. It differs from previous work regarding the tripolar vortex, as obtained in laboratory experiments, in the fact that the laboratory tripole is always obtained from the growth and saturation of an instability in an isolated (zero total circulation) vortex. Here, the flow has total circulation equal to 1.0 , and the amplitude of the initial nonaxisymmetric component determines the strength of the satellites relative to the vortex core. Thus, we can obtain a family of nonshielded tripoles, with satellites of varying size. We have determined a possible threshold perturbation amplitude (acknowledging a degree of arbitrariness in the definition of what constitutes the threshold), for different Reynolds numbers. The critical amplitude is found not to exhibit a power law relationship with Reynolds number, and thus contradicts theoretical results by Ref. 36. We think one possible reason for the disagreement is the considerable nonlinear effects present in the flow, and neglected in the critical layer approach of Ref. 36. Perhaps the nonaxisymmetric vortices of Ref. 36 are entirely different solutions from the tripolar vortices studied here. We have explored the signature of nonlinear effects by calculating the first few azimuthal modes of the vorticity field, at different times in the evolution. This reveals that there is a constant exchange of energy to and from the axisymmetric component, mostly evident for the large perturbations. For the smaller perturbations, one sees also the 2-mode being expelled to larger radii with time, in a similar way to shear-diffusion effects. With regard to the different time scales of the evolution of the flow under study, we find that for small perturbations, the decay scales reasonably well with shear-diffusion time scale, whereas for larger amplitudes only a viscous time scale is observed. Finally, we tested the robustness of this new tripole with nonzero total circulation, and find that it is a very stable structure, recovering from random perturbations, and surviving for many turn-over periods.

\section{ACKNOWLEDGMENTS}

Computing time provided by the Laboratory for Advanced Computation in the Mathematical Sciences (LACMS) of the University of Bristol (http:// lacms.maths.bris.ac.uk/). Thanks to the PETSc team for prompt and always helpful tech support. L.A.B. thanks S. Le Dizès and E. Villermaux for discussions and correspondence. L.A.B.'s travel was possible thanks to a Nuffield Foundation Award.

${ }^{1}$ J. C. McWilliams, "The emergence of isolated coherent vortices in turbulent flow," J. Fluid Mech. 146, 21 (1984).

${ }^{2}$ M. V. Melander, J. C. McWilliams, and N. J. Zabusky, "Axisymmetrization and vorticity-gradient intensification of an isolated two-dimensional vortex through filamentation," J. Fluid Mech. 178, 137 (1987).

${ }^{3}$ Hydrodynamics, sixth ed., edited by H. Lamb (Cambridge University Press, Cambridge, 1932).

${ }^{4}$ D. G. Dritschel, "Nonlinear stability bounds for inviscid, two-dimensional, parallel or circular flows with monotonic vorticity, and the analogous quasi-geostrophic flows," J. Fluid Mech. 191, 575 (1988).

${ }^{5}$ A. J. Bernoff and J. F. Lingevitch, "Rapid relaxation of an axisymmetric vortex," Phys. Fluids 6, 3717 (1994).

${ }^{6} \mathrm{P}$. B. Rhines and W. R. Young, "How rapidly is a passive scalar mixed within closed streamlines?" J. Fluid Mech. 133, 133 (1983).

${ }^{7}$ T. S. Lundgren, "Strained spiral vortex model for turbulent fine structures," Phys. Fluids 25, 2193 (1982).

${ }^{8}$ A. P. Bassom and A. D. Gilbert, "The spiral wind-up of vorticity in an inviscid planar vortex," J. Fluid Mech. 371, 109 (1998).

${ }^{9}$ L. F. Rossi, J. F. Lingevitch, and A. J. Bernoff, "Quasi-steady monopole and tripole attractors for relaxing vortices," Phys. Fluids 9, 2329 (1997).

${ }^{10}$ D. G. Dritschel, "Contour dynamics and contour surgery: Numerical algorithms for extended, high-resolution modelling of vortex dynamics in twodimensional, inviscid, incompressible flows," Comput. Phys. Rep. 10, 77 (1989).

${ }^{11}$ C. F. Driscoll and K. S. Fine, "Experiments on vortex dynamics in pure electron plasmas," Phys. Fluids B 2, 1359 (1990).

${ }^{12}$ P. Koumoutsakos, "Inviscid axisymmetrization of an elliptical vortex," J. Comput. Phys. 138, 821 (1997).

${ }^{13}$ D. G. Dritschel, "On the persistence of non-axisymmetric vortices in inviscid two-dimensional flows," J. Fluid Mech. 371, 141 (1998).

${ }^{14}$ D. G. Dritschel, "Strain-induced vortex stripping," in Mathematical Aspects of Vortex Dynamics (SIAM, Philadelphia, 1989), pp. 107-119.

${ }^{15}$ B. Legras and D. G. Dritschel, "Vortex stripping and the generation of 
high vorticity gradients in two-dimensional flows," Appl. Sci. Res. 51, 445 (1993).

${ }^{16}$ N. J. Zabusky, M. H. Hughes, and K. V. Roberts, "Contour dynamics for the EJuler equations in two dimensions," J. Comput. Phys. 30, 96 (1979).

${ }^{17}$ D. G. Dritschel, "Contour surgery, a topological reconnection scheme for extended interactions using contour dynamics," J. Comput. Phys. 77, 240 (1988).

${ }^{18}$ J. Jiménez, "Hyperviscous vortices," J. Fluid Mech. 279, 169 (1994).

${ }^{19}$ A. Mariotti, B. Legras, and D. G. Dritschel, "Vortex stripping and the erosion of coherent structures in two-dimensional flows," Phys. Fluids 6, 3954 (1994).

${ }^{20}$ G.-H. Cottet and P. Koumoutsakos, Vortex Methods. Theory and Practice (Cambridge University Press, Cambridge, 2000).

${ }^{21}$ L. F. Rossi, "Resurrecting core spreading vortex methods: A new scheme that is both deterministic and convergent," SIAM J. Sci. Comput. (USA) 17, 370 (1996).

${ }^{22}$ L. A. Barba, "Vortex method for computing high-Reynolds number flows: Increased accuracy with a fully mesh-less formulation," Ph.D. thesis, California Institute of Technology, 2004

${ }^{23}$ L. A. Barba, A. Leonard, and C. B. Allen, "Advances in viscous vortex methods-meshless spatial adaption based on radial basis function interpolation," Int. J. Numer. Methods Fluids 47, 387 (2005).

${ }^{24}$ S. Balay, K. Buschelman, W. D. Gropp, D. Kaushik, M. Knepley, L. Curfman-McInnes, B. F. Smith, and H. Zhang, "PETSc User's Manual," Technical Report ANL-95/11-Revision 2.1.5, Argonne National Laboratory, 2002.

${ }^{25} \mathrm{R}$. Franke, "Scattered data interpolation: Tests of some methods," Math. Comput. 38, 181 (1982)

${ }^{26}$ M. D. Buhmann, "Radial basis functions," Acta Numerica 9, 1 (2000).
${ }^{27}$ M. D. Buhmann, Radial Basis Functions. Theory and Implementations (Cambridge University Press, Cambridge, 2003).

${ }^{28}$ R. K. Beatson, J. B. Cherrie, and C. T. Mouat, "Fast fitting of radial basis functions: Methods based on preconditioned GMRES iteration," Adv. Comput. Math. 11, 253 (1999).

${ }^{29}$ L. A. Barba, "Computing high-Reynolds number vortical flows: a highly accurate method with a fully meshless formulation," in Parallel Computational Fluid Dynamics-Multidisciplinary Applications (Elsevier, New York, 2005), pp. 305-312.

${ }^{30}$ G. J. F. van Heijst and R. C. Kloosterziel, "Tripolar vortices in a rotating fluid," Nature (London) 338, 569 (1989).

${ }^{31}$ R. D. Pingree and B. Le Cann, "Three anticyclonic slope water oceanic eddies (swoddies) in the southern Bay of Biscay in 1990," Deep-Sea Res., Part A 39, 1147 (1992)

${ }^{32}$ B. Legras, P. Santangelo, and R. Benzi, "High-resolution numerical experiments for forced two-dimensional turbulence," Europhys. Lett. 5, 37 (1988).

${ }^{33}$ X. J. Carton, G. R. Flierl, and L. M. Polvani, "The generation of tripoles from unstable axisymmetric isolated vortex structures," Europhys. Lett. 9, 339 (1989).

${ }^{34}$ G. J. F. van Heijst, R. C. Kloosterziel, and C. W. M. Williams, "Laboratory experiments on the tripolar vortex in a rotating fluid," J. Fluid Mech. 225, 301 (1991)

${ }^{35}$ K. Bajer, A. P. Bassom, and A. D. Gilbert, "Accelerated diffusion in the centre of a vortex," J. Fluid Mech. 437, 395 (2001).

${ }^{36}$ S. Le Dizès, "Non-axisymmetric vortices in two-dimensional flows," J. Fluid Mech. 406, 175 (2000).

${ }^{37}$ P. Meunier and E. Villermaux, "How vortices mix," J. Fluid Mech. 476, $213(2003)$ 